\title{
Nanoparticle-facilitated functional and molecular imaging for the early detection of cancer
}

\author{
Maharajan Sivasubramanian' ${ }^{1}$ Yu Hsia ${ }^{1,2}$ and Leu-Wei Lo ${ }^{1 *}$ \\ ${ }^{1}$ Institute of Biomedical Engineering and Nanomedicine, National Health Research Institutes, Zhunan, Taiwan \\ 2 Institute of Biotechnology, National Tsing Hua University, Hsinchu, Taiwan
}

\section{Edited by:}

Hidekazu Suzuki, Keio University

School of Medicine, Japan

Reviewed by:

Yoshimasa Saito, Keio University

Faculty of Pharmacy, Japan

Takako Osaki, Kyorin University, Japan

*Correspondence:

Leu-Wei Lo, Institute of Biomedical Engineering and Nanomedicine,

National Health Research Institutes, 35 Keyan Road, Zhunan 350, Taiwan e-mail:Iwlo@nhri.org.tw
Cancer detection in its early stages is imperative for effective cancer treatment and patient survival. In recent years, biomedical imaging techniques, such as magnetic resonance imaging, computed tomography and ultrasound have been greatly developed and have served pivotal roles in clinical cancer management. Molecular imaging (MI) is a non-invasive imaging technique that monitors biological processes at the cellular and sub-cellular levels. To achieve these goals, MI uses targeted imaging agents that can bind targets of interest with high specificity and report on associated abnormalities, a task that cannot be performed by conventional imaging techniques. In this respect, $\mathrm{Ml}$ holds great promise as a potential therapeutic tool for the early diagnosis of cancer. Nevertheless, the clinical applications of targeted imaging agents are limited due to their inability to overcome biological barriers inside the body. The use of nanoparticles has made it possible to overcome these limitations. Hence, nanoparticles have been the subject of a great deal of recent studies. Therefore, developing nanoparticle-based imaging agents that can target tumors via active or passive targeting mechanisms is desirable. This review focuses on the applications of various functionalized nanoparticle-based imaging agents used in $\mathrm{Ml}$ for the early detection of cancer.

Keywords: nanoparticle, molecular imaging, cancer early diagnostics, iron oxide nanoparticles, gadolinium, quantum dots, polymeric nanoparticles, gold nanoparticles

\section{INTRODUCTION}

Cancer is one of the world's most debilitating diseases, and its mortality rates continue to increase. More than 16 million new cancer cases are registered every year, and the medical expenses for overall clinical cancer treatment are practically unaffordable for most patients (Jemal et al., 2011; Morgan et al., 2011; Siegel et al., 2014). These increases in cancer mortality rates are due to a lack of early detection and subsequent treatment. Biomedical imaging techniques, such as magnetic resonance imaging (MRI), optical imaging (OI), X-ray computed tomography (CT) and ultrasound play crucial roles in clinical oncology (Weissleder, 2002; Juweid and Cheson, 2006; Fass, 2008) (Table 1). These imaging methods can provide essential information regarding tumor location, size, and distribution at high spatial resolution. The information obtained from these imaging modalities greatly assists clinicians in treatment strategy selection, which is beneficial for patients. Unfortunately, conventional biomedical imaging techniques detect cancerous tumors when they are more than a centimeter in diameter; at this stage, the lesion already consists of more than $10^{9}$ cells. Here, molecular imaging (MI) is likely to serve a pivotal role because it enables visualization of important biological processes that are associated with the early stages of carcinogenesis in a detailed and specific manner using targeted imaging agents. $\mathrm{MI}$ is more sophisticated than conventional bioimaging because the contrast agents consist of tumor-specific ligands, such as antibodies or peptides. These agents may also be small molecules or signaling moieties, such as fluorophores or radionuclides, or paramagnetic metal chelates. A typical contrast agent is expected to accumulate selectively at a desired site and interact physically or chemically with a target, thereby altering the imaging contrast. Hence, MI serves an integral role in the early detection of cancer and in drug discovery and development (Rudin and Weissleder, 2003; Jaffer and Weissleder, 2005; Weissleder, 2006; Willmann et al., 2008; Pysz et al., 2010; Hussain and Nguyen, 2014).

In general, targeted organic fluorophores and radioisotopes are commonly used as contrast agents in MI (Ballou et al., 1995; Becker et al., 2001; Massoud and Gambhir, 2003; Pham et al., 2004; Schnell et al., 2009). Contrast agents must possess long circulation times and the ability to reach the desired target site at an adequate concentration, which is highly essential for their successful detection in vivo. Unfortunately, clinical applications of contrast agents are severely limited due to their short half-lives, their ability to elicit an immune response and the difficulty that they experience with crossing biological membranes. As a result, only a few imaging agents (For example, Feridex ${ }^{\circledR}$, Magnevist ${ }^{\circledR}$,

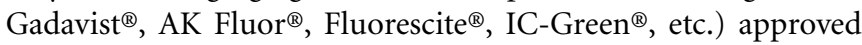
by Food and Drug Administration (FDA, USA) are available for clinical use.

In recent years, nanoparticles have gained significant attention as contrast agents for the early detection of cancer (Figure 1). Using nanoparticles, it is possible to (1) achieve high specificity toward a target, thereby eliminating the danger of side effects; (2) deliver a large payload amount in a single dose and (3) 


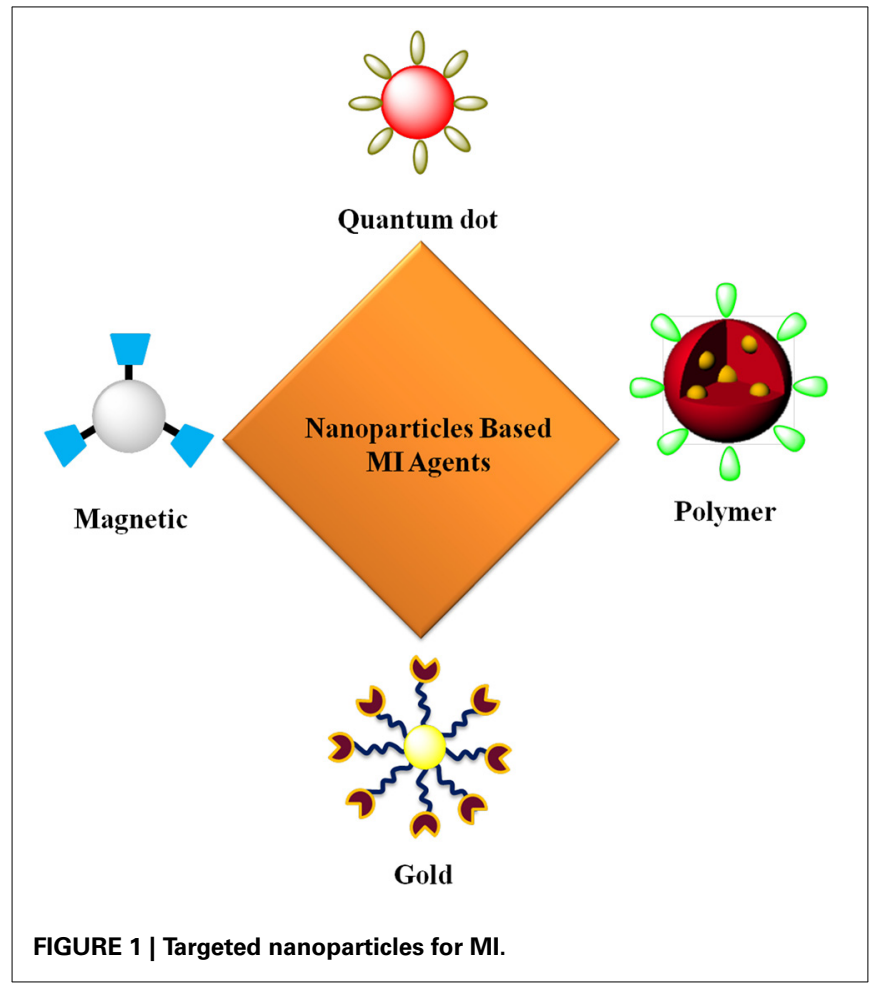

simultaneously deliver both imaging agents and therapeutics. Carefully designed nanoparticle-based contrast agents (NCAs) could overcome biological barriers and reach tumors through either active or passive targeting mechanisms (LaVan et al., 2003; Ferrari, 2005; Couvreur and Vauthier, 2006; Peer et al., 2007) (Figure 2). In passive targeting, a distinguishing feature of a tumor enables an NCA to accumulate and be retained in the tumor via the enhanced permeation and retention (EPR) effect. In contrast, active targeting involves the conjugation of targeting ligands to the surface of the NCA, enabling the NCA to enter the cancer cell via receptor-mediated endocytosis (Saravanakumar et al., 2009; Choi et al., 2010; McCarthy et al., 2010). The aim of this review is to highlight various NCAs used for early cancer detection with MI.

\section{MRI}

MRI uses the nuclear magnetic resonance signals of hydrogen atoms in water to generate images. After a brief radiofrequency pulse, the rate at which protons relax back to their equilibrium state, dependent on their physiochemical environment, gives rise to an MRI signal. MRI holds great potential as a clinical imaging and diagnostic tool due to its excellent soft tissue contrast, high spatial resolution, and ability to deeply penetrate tissues (Stark and Bradley, 1999; Jacobs and Cherry, 2001; Sosnovik and Weissleder, 2007). The most commonly used contrast agents $\left(\mathrm{T}_{1}\right.$ and $\mathrm{T}_{2}$ ) for clinical cancer diagnosis are gadolinium chelates and superparamagnetic iron oxide nanoparticles (SPIOs) (McCarthy and Weissleder, 2008; Islam and Josephson, 2009; Lin et al., 2009; Cheng et al., 2013; Zhou and Lu, 2013). Bare SPIOs are used in the clinic to image liver tumors and metastases with sharp contrast, providing clear distinction between normal and cancerous tissues.

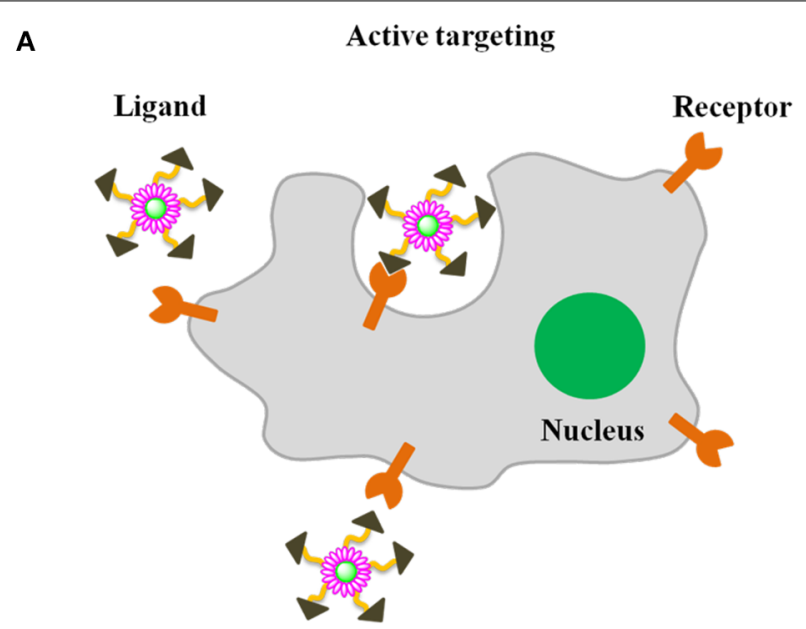

B Passive targeting

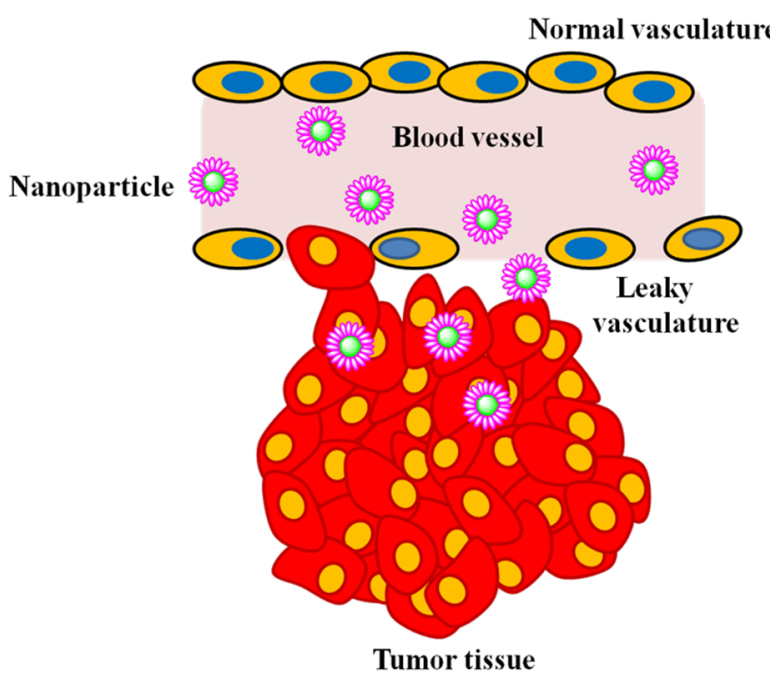

FIGURE 2 | Illustration of mechanism of tumor targeting (A) active targeting and (B) passive targeting.

This diagnostic ability arises from the fact that SPIOs are readily taken up by reticuloendothelial cells (Kupffer cells), which are absent from or present in lower concentrations in tumors (Saini et al., 1987; Lin et al., 2009). SPIOs with diameters of $30 \mathrm{~nm}$ are known to accumulate in lymph nodes by interstitial-lymphatic fluid transport and can be consequently used to detect lymph node metastases (Anzai et al., 2003). In addition, ultrasmall SPIOs (USPIOs) have been investigated as contrast agents for improving the delineation of brain tumors on MR images (Enochs et al., 1999; Neuwelt et al., 2004).

\section{IRON OXIDE NANOPARTICLES}

To improve tumor detection and localization, SPIOs are chemically modified with ligand molecules that target specific upregulated receptors or proteins and serve as MI probes. Yang et al. developed functionalized iron oxide nanoparticles (IOs) to target urokinase-type plasminogen activator receptor (uPAR). uPAR 
is well known to be upregulated in a variety of tumor cells and tumor-associated endothelial cells, such as fibroblasts and macrophages (Han et al., 2005). High uPAR expression is closely associated with metastasis and tumor invasiveness; hence, uPAR is an excellent target for imaging breast cancer cells. In this study by Yang et al., IOs were coated with a carboxylic acid-bearing amphiphilic polymer. The large number of carboxylic groups on the surface allowed covalent modification with poly(ethylene glycol) (PEG) and uPA bearing Cy5.5. Confocal microscopy experiments demonstrated effective uptake of the targeted nanoparticles by $\mathrm{uPA}$ receptors, which were overexpressed on mouse mammary tumor 4T1 cells, whereas human breast cancer T47D cells that lacked uPA receptors did not uptake the nanoparticles. Biodistribution studies in 4T1 tumor-bearing mice demonstrated significant accumulation of the nanoagent in the tumors and showed increased fluorescence signal intensity for up to $48 \mathrm{~h}$. Similar tumor-targeting properties of these nanoagents were also observed using MR imaging in a mouse model (Yang et al., 2008). In another study, Yang et al. used the amino-terminal fragment (ATF) of the high affinity receptor binding domain of uPAconjugated IOs to target UPAR. The results of this study showed that ATF-conjugated IO specifically bound to UPAR and was taken up by the cells. In vivo systemic delivery of ATF-IO nanoparticles showed selective accumulation in orthotopic pancreatic tumors, as demonstrated by both $\mathrm{OI} / \mathrm{MR}$ imaging. In vivo OI clearly showed strong near infrared signals in the pancreatic tumors; however, no signal was detected in the liver or spleen of the tumor-bearing mice that received Cy5.5-ATF-IO nanoparticles. Additionally, the targeted nanoparticles exhibited high sensitivity and were able to detect tumors as small as $0.5-1 \mathrm{~mm}^{3}$ in animal tumor models using MRI. The authors suggested that the targeted nanoparticles used in this study have the potential for early detection of pancreatic cancer lesions by MRI and for intraoperative delineation of tumor margins and peritoneal metastases by OI (Yang et al., 2009a).

Chen et al. developed herceptin (HP)-conjugated cross-linked iron oxide nanoparticles (CLIOs) for breast cancer imaging. HP is a monoclonal antibody that has the ability to bind HER-2/neu receptors, which are overexpressed in breast cancer cells (Tran et al., 2007). Primary amine-functionalized CLIOs were prepared by cross-linking dextran-coated SPIOs with 2,2' (ethylenedioxy)bisethylamine, followed by chemical conjugation with HP. In vitro studies demonstrated efficient uptake of targeted CLIOs in HER-2/neu receptor-overexpressing cancer cells (SKBr3, MDA-MB-231, and MCF-7); uptake was not observed in $\mathrm{KB}$ cells that lacked HER-2/neu receptors. From in vivo MRI studies, the results showed that when administered intravenously in mice bearing SKBr3 and KB tumors, targeted CLIOs selectively accumulated in the SKBr3 tumors but not in the KB tumors, as confirmed by the darkening of the tumors in $\mathrm{T}_{2}$-weighted images (Chen et al., 2009). Li et al. developed octreotide peptide (OP)modified USPIOs to target somatostatin receptors (SSR) in breast cancer cells. Somatostatin (ST), a polypeptide known as somatotropin release inhibiting factor (SRIF), exhibits an affinity for the receptors overexpressed in neuroendocrine tumors and on cells of the immune system. OP is an analog of ST that has the ability to bind several SSR subtypes. To prepare SSR-targeted contrast agents, one end of dicarboxylated PEG was attached to USPIOs, and the other end was conjugated to OPs. In vitro MR imaging results revealed that the targeted nanoparticles displayed significantly lower $\mathrm{T}_{2}$ signal values, suggesting efficient uptake by breast carcinoma MCF-7 cells compared to non-targeted nanoparticles. For in vivo MR imaging studies, both targeted and non-targeted nanoparticles were administered to MCF-7 tumorbearing mice. The results showed that the targeted nanoparticles accumulated in the tumor, which was confirmed by the observed signal decrease in $\mathrm{T}_{2}$-weighted images. In addition, non-targeted nanoparticles also exhibited a small decrease in signal; this might be due to random accumulation in the tumor by the EPR effect ( $\mathrm{Li}$ et al., 2009). Luteinizing hormone-releasing hormone (LHRH) is known to bind LHRH receptors that are overexpressed on breast cancer cell membranes (Leuschner et al., 2005). Hence, LHRH is a suitable breast tumor-targeting molecule. Meng et al. developed LHRH receptor-targeted SPIOs to image breast tumors. In vitro and in vivo studies in breast cancer cells demonstrated the use of targeted nanoparticles as tumor-specific $\mathrm{T}_{2}$ contrast agents in relevant models. The cell uptake behavior of targeted SPIOs was much greater than that of bare SPIOs. The increased uptake and intracellular accumulation of targeted SPIOs provided enhanced $\mathrm{T}_{2}$ contrast with improved spatial resolution. The improved targeting by this nanoagent may also be used for cancer therapy via loading the nanoparticles with therapeutic drugs (Meng et al., 2009).

Kelly et al. developed a new peptide (IPL) to image prostate cancer. IPL has the ability to bind a type II transmembrane serine protease called hepsin $(\mathrm{HPN})$, which is uniquely expressed in prostate cancer cells. In this study, IPL was conjugated to a bimodal nanoparticle that bore an optically detectable fluorophore attached to IOs. In a mouse xenograft model, HPN-targeted nanoparticles exhibited high specificity for HPN-overexpressing LNCaP xenografts compared to non-HPNexpressing xenografts. The authors proposed that HPN-targeted nanoparticles could be used for the early detection of prostate cancer (Kelly et al., 2008). Kresse et al. developed transferrin (Tf)-conjugated USPIOs for targeting malignant tumors. Tf is an iron-transporting serum glycoprotein that has the ability to bind specific receptors that are overexpressed in various cancer cells (Hopkins and Trowbridge, 1983). In vivo MR imaging studies in SMT/2A tumor-bearing rats showed that targeted USPIOs exhibited substantial signal reduction (40\%) in $\mathrm{T}_{2}$-weighted $\mathrm{MR}$ images. In addition, control experiments using bare USPIOs or non-specific human serum albumin-conjugated USPIOs also accumulated in the tumors with a $10 \%$ change in the MR signal intensity, most likely due to non-specific accumulation in the tumors (Kresse et al., 1998). Choi et al. used folic acid (FA)conjugated magneto-fluorescent nanoparticles as a targeted imaging agent for cancer detection. FA is essential for cell proliferation and for the maintenance of new cells during rapid cell division. Folate receptors are frequently upregulated in various cancer cells, enabling cancer cells to access vitamins necessary for their survival (Antony, 1996; Lu and Low, 2002). Hence, folate-targeted imaging probes are useful for the detection of cancer cells that overexpress folate receptors. In vitro confocal observation showed a strong fluorescence signal in folate receptor-overexpressing $\mathrm{KB}$ 
cells incubated with the targeted nanoagent, whereas no fluorescence signals were detected in cells (human lung carcinoma) lacking folate receptors. An in vivo MR imaging study in a mouse $\mathrm{KB}$ tumor xenograft model showed that the targeted nanoagent exhibited an average intensity decrease of $38 \%$ from preto post-contrast images of the tumor, which was approximately three times the intensity decrease observed in a non-tumorbearingmouse. These targeted nanoparticles are highly specific and can be used as targeted delivery systems for tumor diagnosis and therapy (Choi et al., 2004).

For targeting brain tumors, Meng et al. developed SPIOFCs, a dual imaging nanoparticle consisted of chlorotoxin (CTX) and fluorescein isothiocyanate (FITC) conjugated to SPIOs. CTX, a small peptide found in the deathstalker scorpion (Leiurus quinquestriatus), is a highly specific marker for glioma cells and has been used for brain tumor targeting in animal models (Soroceanu et al., 1998). In vitro studies demonstrated that prepared SPIOFCs did not induce any toxicity and showed increased cellular uptake in human U251-MG and rat C6 glioma cells when evaluated by MR imaging and inductively coupled plasma emission spectroscopy (ICPMS). Glioma cells were found to be insensitive to bare nanoparticles without CTX and showed no significant uptake; these findings suggested that the presence of CTX was essential for specific and efficient targeting of glioma cells (Meng et al., 2007). Another study by Sun et al. used CTX-conjugated PEGylated iron oxide nanoparticles (CPIs) as an MI probe to detect gliomas. In general, the presence of PEG in nanoparticles offers stealth properties and allows the conjugation of various molecules, such as diagnostic, targeting, and therapeutic agents. In vitro MR imaging showed increased cellular uptake of CPI nanoparticles in 9L gliosarcoma cells compared to non-targeted nanoparticles. In athymic (nu/nu) mice bearing 9L gliosarcoma xenograft tumors, CPIs gradually accumulated in the tumors over time as expected, and small amounts of non-targeted nanoparticles also reached the tumors through non-specific mechanisms. The contrast enhancement degree was quantified by measuring the $\mathrm{R}_{2}$ relaxation rates (Sun et al., 2008). For the specific identification of apoptotic cells, Schellenberger et al. chemically conjugated CLIOs to annexin-V, a protein that binds to extracellular phosphatidylserine, an early marker of apoptosis (Martin et al., 1995). In this study, Jurkat T lymphoma cancer cells were used, it is well known that insufficient apoptosis is one of the characteristic features of cancer cells. For specific identification, apoptosis was induced in Jukart cells using camptothecin, an anticancer drug. From in vitro MRI studies, it was found that targeted CLIOs identified camptothecin-induced apoptosis of Jurkat T cells, which was confirmed by the significant signal decrease relative to untreated cells; in contrast, bare CLIOs failed to induce significant changes in apoptotic cells. These results suggest that the targeted CLIOs used in this study can serve as an MRI probe for imaging apoptosis in vivo (Schellenberger et al., 2002).

\section{Clearance of IOs}

In general, intravenously administered IOs are cleared from blood circulation by reticulo endothelial system (Corot et al., 2006). However, the various properties such as shape, size, and surface characteristics largely determine the pharmacokinetics and clearance. For instance, IOs with larger size are eliminated faster than smaller-sized NPs. Jain et al. investigated the biodistribution, clearance, and biocompatibility of IOs sequentially coated with oleic acid and pluronic (Pluronic-OA-IO). Usually, IO formulations use dextran or starch coating as a stabilizing agent to form a water-dispersible system. In this study, pluronic or PEG were used as coating materials, which are known to prevent opsonization. In vivo biodistribution studies of Pluronic-OA-IO (193 nm) showed about $55 \%$ of the injected iron accumulated in the liver. A gradual increase in the serum iron level was observed for 1 week, which indicated that clearance of nanoparticles require more than 3 weeks (Jain et al., 2008).

Lewis et al. studied the biodistribution of Ferumoxtron-10, a USPIO ( $30 \mathrm{~nm}$ in diameter) coated with low molecular weight dextran. Ferumoxtran-10 demonstrated slow clearance of iron and was eliminated (89\%) in the urine in 7 weeks. Iron contained in Ferumoxtron-10 was absorbed into the body's iron store and gradually into hemoglobin. Like endogeneous iron, it was eliminated slowly (16-21\%) after 84 days through hepatobiliary mechanism (Bourrinet et al., 2006).

\section{GADOLINIUM-BASED NANOPARTICLES}

Paramagnetic gadolinium (Gd) complexes are the most widely used $\mathrm{T}_{1}$ contrast agents for clinical applications of MRI (Caravan et al., 1999; Lee et al., 2014). In recent years, many researchers have investigated the use of several targeted Gd complexes for the early detection of cancer (Damadian, 1971; Zhou and Lu, 2013; Hussain and Nguyen, 2014). The use of Gd complexes as positive contrast agents in $\mathrm{T}_{1}$-weighed $\mathrm{MRI}$ sequences is limited by their sensitivity. Hence, Gd complexes are usually encapsulated in nanocarriers or are chemically conjugated to synthetic polymers, such as dendrimers, to amplify their sensitivity (Kobayashi and Brechbiel, 2003, 2005; Luo et al., 2011). For targeting purposes, Gd complexes are covalently conjugated to targeted ligands, which dock and respond to specific receptors that are overexpressed in cancer cells.

Dendrimers are self-assembled polymer constructs with tunable surface and are widely used as carriers for Gd-based contrast agents. $\mathrm{Xu}$ et al. developed a bimodal second-generation (G2) dendrimer nanoparticle that consisted of covalently conjugated Gd-1B4M- diethylenetriaminepentaacetate (DTPA) and optically detectable rhodamine green for targeting ovarian cancer (Figure 3). The nanoparticles achieved success in reaching tumor tissues and delivered significant amounts of imaging payloads in an ovarian tumor-bearing mouse xenograft model to produce detectable changes in the tumors by optical and MRI (Xu et al., 2007). Regino et al. evaluated the use of the dendrimerbased contrast agent Gd-G8 as a dual MRI and CT contrast agent for precisely monitoring convection-enhanced delivery to the brain. From in vitro studies, CT-based attenuation values of the Gd-based agent were $\sim 1.6$ times greater than those of iopamidol (an iodine-based agent); moreover, the attenuation of the Gd-G8 dendrimer was similar to that of Gd-DTPA. In vivo studies demonstrated that detectable signal enhancement could be observed by CT and MRI with an optimal dose of Gd-G8 over a range of $23-78 \mathrm{mM}$; however, for the adequate delineation of the injection site on MRI and CT, a concentration of at least 


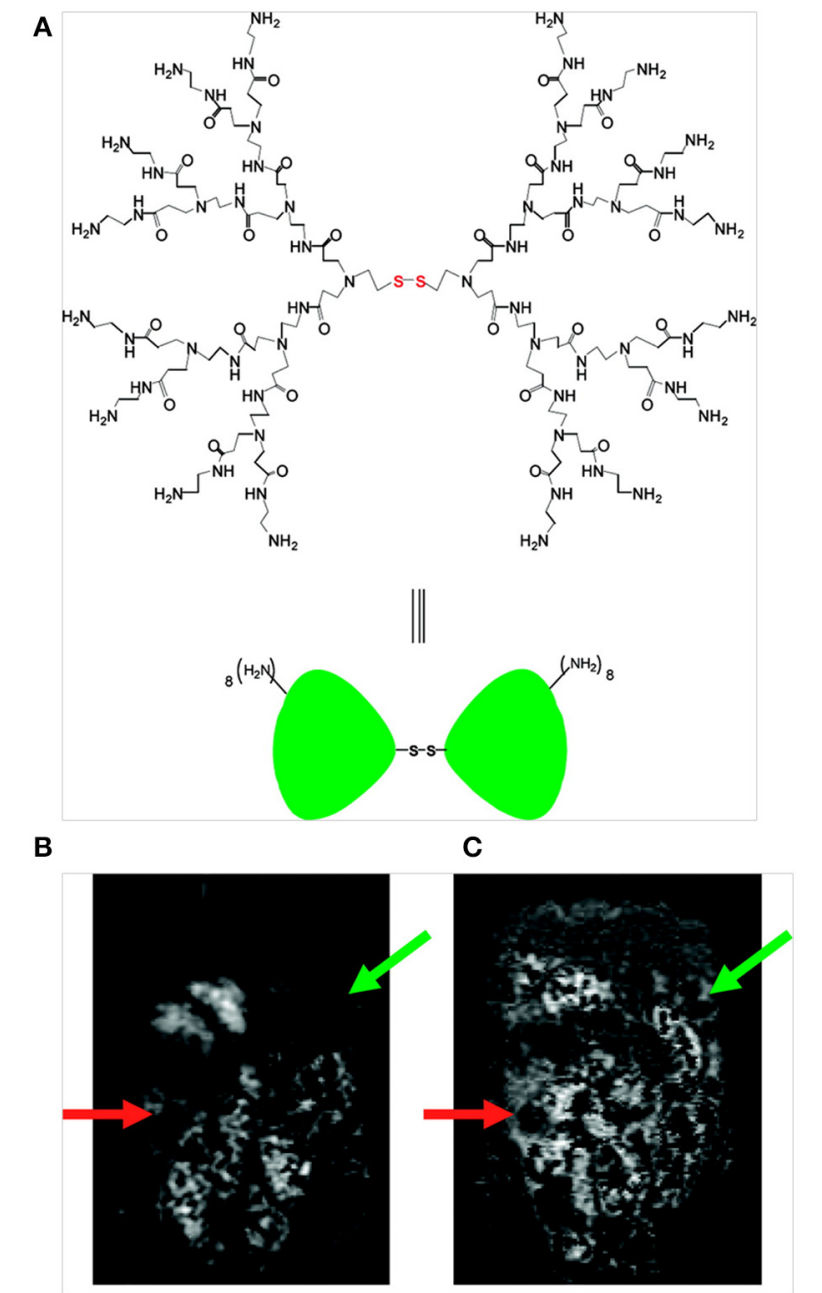

FIGURE 3 | (A) Structure of a PAMAM dendrimer possessing a disulfide cystamine core; (B) $\mathrm{T}_{1}$-weighed MR image obtained prior to contrast agent injection $(t=0)$ reveals an area of tumor growth below the liver, stomach and spleen in the left abdomen (green arrow) and adjacent to the gastrointestinal tract in the lower right abdomen (orange arrow) and (C) $\mathrm{T}_{1}$-weighted MR image obtained $6.5 \mathrm{~h}$ after intra-peritoneal injection of the dual contrast agent (green and orange arrows indicate signal enhancement on the tumor surface) (Xu et al., 2007), (C) 2007, American Chemical Society.

$47 \mathrm{mM}$ was required, and the distribution volume $(\mathrm{Vd})$ was estimated with greater sensitivity by MRI than by CT (Regino et al., 2008).

Swanson et al. prepared a target-specific multifunctional nanoparticle, Gd(III)-DOTA-G5-FA that consisted of Gdcomplexed, 1,4,7,10-tetraazacyclododecane-1,4,7,10-tetraacetic acid (DOTA) and FA conjugated to a G5 poly (amidoamine) (PAMAM) dendrimer. In vivo studies were performed in a NODCB-17 SCID mouse xenograft model with KB tumors. When administered subcutaneously, the Gd(III)-DOTA-G5-FA dendrimer nanoparticles specifically reached the tumors and generated statistically significant MRI signal enhancement compared to non-targeted counterparts (Gd(III)-DOTA-G5). Sustained signal enhancements in the tumors were observed even at $48 \mathrm{~h}$ post-injection, suggesting the effective tumor-targeting ability of Gd(III)-DOTA-G5-FA (Swanson et al., 2008). Huang et al. prepared multifunctional dendrigraft poly-L-lysines (D2 and D3) composed of covalently attached CTX and Gd-DTPA. The OI results showed that CTX dendrimer conjugates were readily taken up by C6 glioma and liver tumor cell lines but were not taken up by normal cell lines. Intracranially administered CTX dendrimer conjugates in C6 glioma-bearing mice preferentially accumulated in the brain tumors, suggesting their tumor-targeting ability. In addition, the MRI results demonstrated substantial signal enhancement in mice treated with CTX-modified contrast agent, which reached a maximum level at $5 \mathrm{~min}$ for both gliomas and liver tumors (Huang et al., 2011).

Olson et al. developed a G5 PAMAM dendrimer coated with activatable cell-penetrating peptides (ACPPs) bearing Cy5 or Gd or both for the dual monitoring of matrix metalloproteinase (MMP) activity. The uptake of ACPP-conjugated nanoparticles in a human fibrosarcoma cell line was 4 - to fifteen-fold higher than that of bare nanoparticles. Residual tumors and metastases as small as $200 \mu \mathrm{m}$ were successfully detected using OI. In the presence of protease, Gd-labeled nanoparticles released high levels of Gd (30-50 $\mu \mathrm{m})$ to the tumor parenchyma, which resulted in enhanced monitoring using $\mathrm{T}_{1}$-weighted contrast for a longer period after injection (Olson et al., 2010).

Inadequacies in the ability to image and treat malignant gliomas are mainly due to the blood-brain barrier. Sarin et al. developed several Gd-DTPA-functionalized PAMAM dendrimers of various sizes for transvascular delivery across the bloodbrain barrier. When administered intravenously in RG-2 gliomabearing mice, Gd- and fluorescent dye-functionalized dendrimers $(11.7-11.9 \mathrm{~nm})$ effectively crossed the blood-brain barrier and accumulated in the tumors. In this study, the authors found that the physiological upper limit of nanoparticle size for traversing the blood-brain barrier was less than 11.7-11.9 nm (Sarin et al., 2008). This investigation also showed that in the physiological state, a fibrous glycocalyx covers the luminal surface of the blood-tumor barrier (BTB) of both the brain tumor and the peripheral tumor microvasculature, which is the major impediment for the transport of particles. Hence, the authors indicated that the peripheral tumor vasculature is highly permeable to macromolecules smaller than approximately $12 \mathrm{~nm}$ due to the presence of a greater number of pores underlying the glycocalyx of the BTB (Huang et al., 2011). Nanoglobular Gd-DOTA conjugates [lysine dendrimers (G2 and G3) with cubic silsesquioxane cores] were attached to the peptide CLTI, which specifically binds to fibrin-fibronectin complexes that are present in the tumor extracellular matrix (Pilch et al., 2006), and were subsequently used for MRI identification of tumor tissues in female nude mice bearing MDA-MB-231 human breast carcinoma xenografts. The $\mathrm{T}_{1}$ relaxivities of the targeted $\mathrm{G} 2$ and $\mathrm{G} 3$ nanoglobules were 7.92 and $8.20 \mathrm{mM}^{-1} \mathrm{~s}^{-1}$, respectively, and the nanoglobules exhibited greater contrast enhancement in tumor models than corresponding non-targeted contrast agents (Tan et al., 2014). In another study, a Gd-DTPA-PAMAM-PEG dendrimer was conjugated to T7, a peptide that has an affinity for Tf receptors expressed on gliomas and liver cancer cells (Han et al., 2010, 2011a). T7-conjugated Gd-DTPA-PAMAM-PEG dendrimers 
(T7- GdDTPA-PAMAM-PEG) were then used for MRI identification of tumors in human hepatocarcinoma Bel-7402- and glioma C6-bearing mice. T7-GdDTPA-PAMAM-PEG exhibited an excellent ability to target tumors in hepatocarcinoma-bearing mice; moreover, the accumulation of T7-GdDTPA-PAMAM-PEG was $162.5 \%$ times that of PAMAM-PEG. However, the peptide conjugate was insensitive to gliomas (Han et al., 2011b). Multifunctional nanoparticles carrying Gd-DTA and FA conjugated to cross-linked G5 PAMAM dendrimers (dendrimer nanoclusters) were developed for tumor-targeted MRI. FITC-labeled dendrimer nanoclusters were readily taken up by folate receptorexpressing KB cancer cells, and in the presence of free FA, cell uptake was significantly reduced, suggesting receptor-mediated uptake. In mice bearing KB xenograft tumors, subcutaneously administered dendrimer nanoclusters gradually accumulated in the tumors; this accumulation was detected with a strong MRI signal. The incorporation of FA in the dendrimer nanoclusters established tumor specificity, which resulted in enhanced images (Cheng et al., 2010).

\section{이}

OI is a non-invasive technique that can be broadly divided into fluorescence and bioluminescence imaging. Fluorescence imaging is based in principle on using external light energy of an appropriate wavelength to excite a fluorophore; the excited fluorophore then emits a light of longer wavelength with lower energy. In contrast, bioluminescence imaging involves a chemical reaction between target and substrate molecules to generate luminescence, which is detected externally as evidence of a molecular process (Luker and Luker, 2008; Weissleder and Pittet, 2008; Willmann et al., 2008). The main challenges to OI are limited tissue penetration and tissue autofluorescence. Light in the visible spectrum is strongly absorbed by hemoglobin and other biomolecules, such as water and lipids, which significantly reduces the signal by ten-fold. As an alternative to light in the visible region of the spectrum, light in the near infrared (NIR) region $(600-1000 \mathrm{~nm})$ has been used to image fluorescence in deeper tissues because tissue autofluorescence is reduced significantly in the NIR region, and little absorption of light by the endogenous biomolecules occurs. Hence, tremendous effort has been invested in the development of NIR probes for OI.

\section{QUANTUM DOTS}

Semiconducting fluorescent nanocrystals known as quantum dots (QDs) have been widely used as probes for biomedical imaging and labeling due to their unique optical properties. QDs exhibit broad absorption spectra, high quantum yields, low photobleaching, and resistance to chemical degradation compared to traditional fluorophores, such as organic dyes (Bruchez et al., 1998; Medintz et al., 2005; Michalet et al., 2005). For cancer-related molecular and cellular imaging applications, the surface of QDs is usually modified with biomolecules, such as antibodies, peptides or small molecules that have the ability to adhere and respond to receptors or antigens present on target cells (Zhang et al., 2008; Lee et al., 2010a; McCarthy et al., 2010; Cheng and Cheng, 2012; Pericleous et al., 2012). Substantial developments in nanotechnology and materials chemistry have encouraged scientists to engineer QDs to carry therapeutic agents for concurrent imaging and therapy.

Epidermal growth factor receptor (EGFR) is a transmembrane glycoprotein that is overexpressed in breast cancers and can be utilized as a biomarker for imaging (Herbst, 2004). Yang et al. developed anti-EGFR antibody-conjugated QDs for targeted breast cancer imaging. Initially, carboxylic acid-functionalized QDs were covalently linked to the primary amine of modified nickel (II) nitrilotriacetic acid (NNTA) via a stable amide bond. To target EGFR, a histidine-tagged anti-EGFR antibody (ScFv EGFR) was conjugated to the NNTA-QDs. In vitro studies clearly demonstrated that the targeted NNTA-QDs exhibited an affinity for EGFR-overexpressing breast cancer cells (4T1 and MDA-MB-231) and were readily internalized, whereas nontargeted NNTA-QDs showed no significant binding to breast cancer cells (Yang et al., 2009b). Gao et al. reported prostatespecific membrane antigen (PSMA)-bearing QD conjugates for targeted in vivo imaging of prostate cancer. Initially, QDs were sequentially coated with n-octylphosphine oxide and a carboxylic acid-bearing amphiphilic polymer. The polymer served to protect the QDs from enzymatic digestion and hydrolysis in vivo. Surfacecoated QDs were then conjugated to PEG to prolong their circulation time in vivo. In vitro studies demonstrated that the targeted QDs were readily taken up by PSMA-overexpressing C4-2 human prostate cancer cells, while non-targeted PEGylated QDs exhibited minimal uptake by prostate cancer cells. When administered to prostate tumor-bearing nude mice, targeted QDs accumulated in the tumors with high specificity and exhibited a strong fluorescence signal, whereas no significant fluorescence signal was observed in mice injected with PEGylated QDs. Further studies, including biodistribution and histological analyses, clearly illustrated the prostate tumor-targeting potential of functionalized QDs (Gao et al., 2004). Bagalkot et al. developed QD-Apt, QDs functionalized with A10 RNA aptamer that recognizes the extracellular domain of PSMA for prostate cancer imaging, therapy and sensing. Doxorubicin (DOX) was able to bind to the doublestranded A10 RNA aptamer via non-covalent interactions, and the resulting conjugate (QD-Apt DOX) gained reversible selfquenching properties based on bi-fluorescence resonance energy transfer (Bi-FRET); in this process, QD-DOX and QD-Apt DOX acted as donor-acceptor pairs (Figure 4). Thus, the fluorescence of the QD-Apt DOX conjugate was quenched when intact, and the release of DOX from QD-Apt DOX inside of the cell allowed the nanoparticles to recover their fluorescence. When incubated with PSMA-overexpressing cancer cells (LNCaP), the nanoparticles were taken up and delivered DOX, which was confirmed by a strong fluorescence signal (Bagalkot et al., 2007).

Scroeder et al. developed lipodots, which consisted of QDs entrapped in a lipid shell and post-loaded with a folate-lipid conjugate for targeting tumors. In vitro confocal observations showed that the targeted lipodots were readily taken up by folate receptoroverexpressing cancer cells (J6456-FR and KB-FR), confirmed by a strong fluorescence signal, while non-targeted lipodots were found to be insensitive. In vivo studies with mice bearing J6456FR tumors demonstrated the tumor-targeting ability of targeted lipodots following intra-peritoneal injection (Schroeder et al., 2007). Mulder et al. developed targeted bimodal nanoparticles 
A

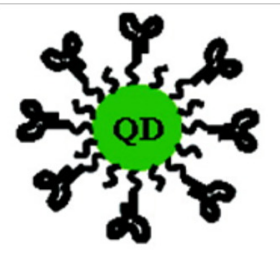

QD-Apt: “ON"
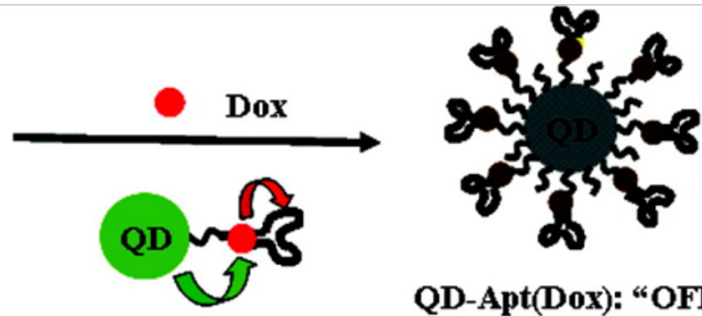

QD-Apt(Dox): “OFF"

B
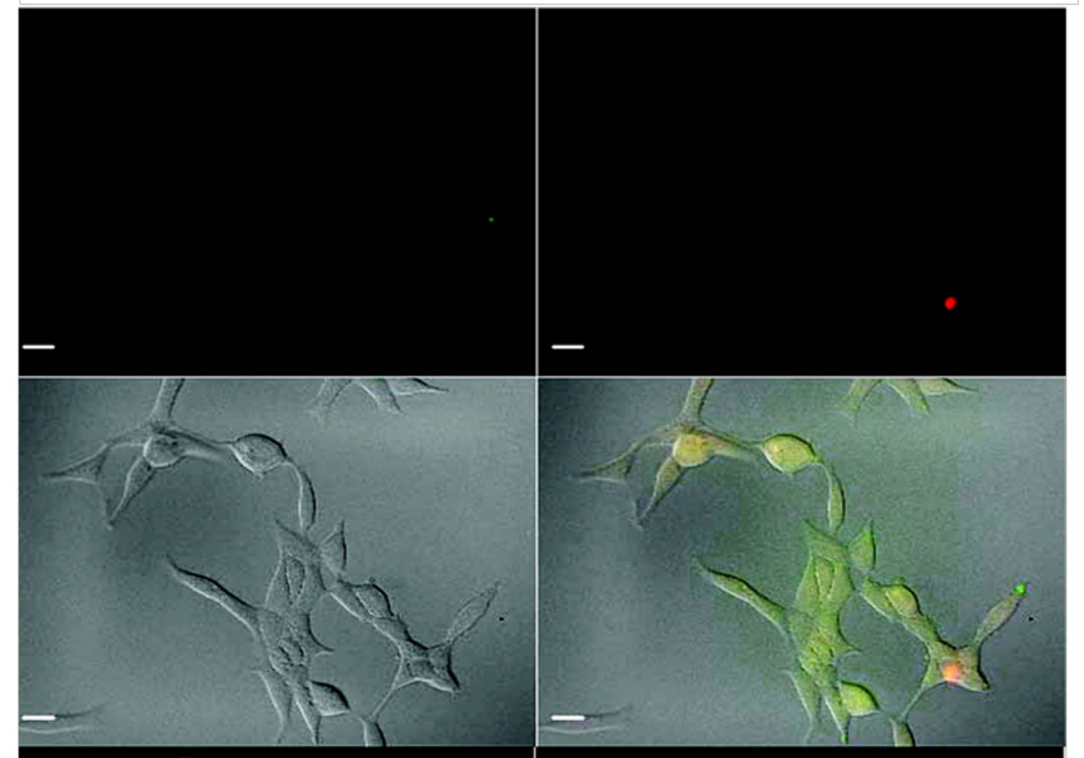

C

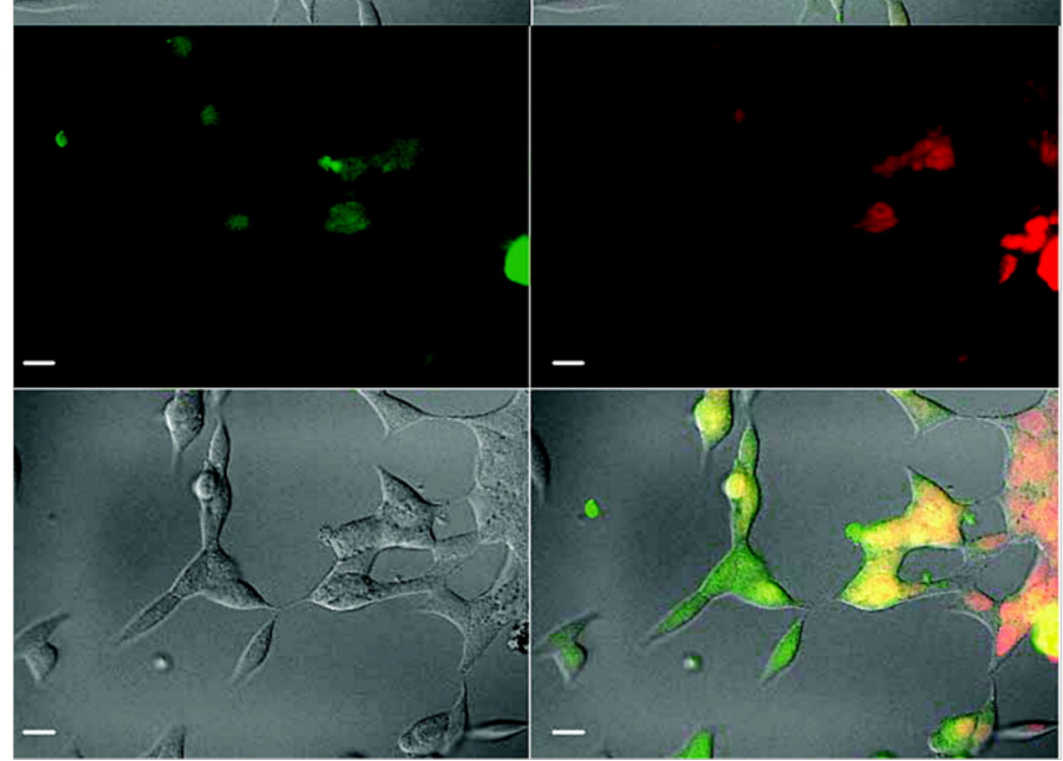

FIGURE 4 | (A) A schematic illustration of QD-Apt(Dox) Bi-FRET. Confocal laser scanning microscopy images of QD-Apt DOX conjugates incubated in PSMA-expressing LNCaP cells at (B) $0 \mathrm{~h}$ and (C) $1.5 \mathrm{~h}$. DOX and QDs are shown in red and green, respectively, and the lower right images in each panel represent an overlay of DOX and QD fluorescence (Bagalkot et al., 2007), (C) 2007, American Chemical Society.
(pQDs) that consisted of magnetically detectable PEGylated lipids that were covalently coupled to QDs bearing RGD (specific for $\alpha_{v} \beta_{3}$ integrin interaction). When incubated with $\alpha_{v} \beta_{3}$ integrin-overexpressing human umbilical vein endothelial cells (HUVECs), enhanced uptake of pQDs by HUVECs was observed compared to that of non-targeted pQDs. The optical properties of the $\mathrm{pQDs}$ were preserved, and they exhibited very high $r 1$ relaxivity. The overall results suggested the ability of the nanoagent to target tumor vasculature (Mulder et al., 2005). In a similar study, Cai et al. evaluated the tumor vasculature-targeting 


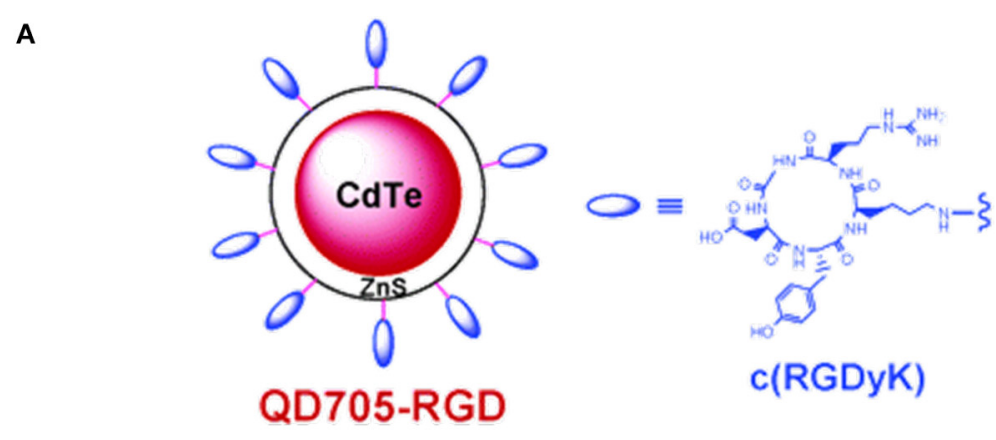

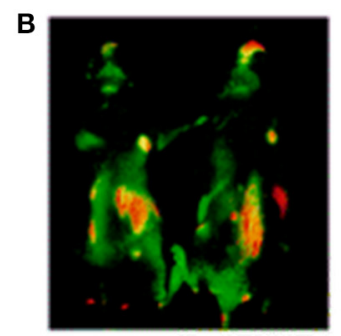

$1 \mathrm{~h}$

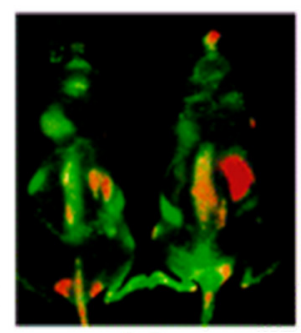

$4 \mathrm{~h}$

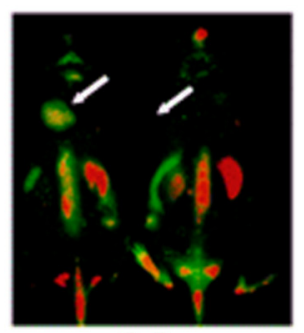

$6 \mathrm{~h}$

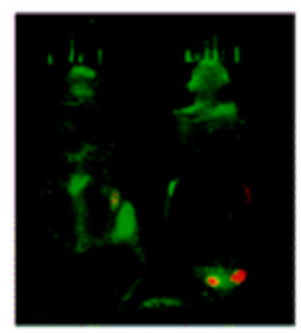

27 h

FIGURE 5 | (A) RGD-bearing QD705 conjugates and (B) in vivo fluorescence imaging of tumor vasculature in a U87MG tumor-bearing mouse at various time intervals (Cai et al., 2006), (C) 2006, American Chemical Society.

ability of RGD-coupled QD 705 (QD705-RGD) (Figure 5). When administered intravenously in athymic nude mice bearing subcutaneous U87MG human glioblastoma tumors, QD705-RGD exhibited a significant ability to target tumor vasculature, and the tumor fluorescence intensity reached a maximum at $6 \mathrm{~h}$ postinjection. However, non-specific accumulation was also observed in the lymph nodes, liver, and bone marrow (Cai et al., 2006). Chang et al. prepared protease-activatable QDs that consisted of gold nanoparticles bound to QDs via a peptide sequence susceptible to degradation by enzyme proteases. When intact, the conjugate was optically quenched with a $71 \%$ reduction in luminescence; however, the conjugate became highly luminescent after the peptide linker was degraded by proteases. This type of probe could be useful for monitoring the biological activities of proteases (Chang et al., 2005). Smith et al. observed and recorded the binding of RGD-conjugated QD800 (QD with emission at approximately $800 \mathrm{~nm}$ ) to tumor blood vessels expressing $\alpha_{v} \beta_{3}$ in mice models. RGD-QD800 demonstrated significant uptake by tumors compared to control QDs (PEG-QD800 and RADQD800), suggesting the ability of RGD-QD800 to target tumors overexpressing $\alpha_{v} \beta_{3}$. Intra-vitalmicroscopy observations in mice bearing an SKOV-3 ear tumor clearly showed that the targeted QDs did not target tumor cells but did target the tumor neovasculature. This observation was presumably due to their relatively large size ( $\sim 20 \mathrm{~nm}$ in hydrodynamic diameter), which prevented the nanoparticles from extravasating into the tumor. The authors also found that RGD-QD800 adhered to their targets as aggregates rather than as individual nanoparticles (Smith et al., 2008). In an interesting work by Akerman et al., QDs were conjugated to three different peptides (GFE, F3, and Lyp-1) to demonstrate their specific targeting abilities. In vivo studies showed that
QD-GFE (lung-targeting) conjugates accumulated in the lungs of mice following intravenous administration, whereas QD-F3 and QD-Lyp-1 conjugates specifically targeted blood vessels or lymphatic vessels in tumors. Unfortunately, the QDs were not sufficiently stable to remain luminescent in living cells and tissues. Hence, the QDs used in this investigation are not feasible for in vivo applications (Akerman et al., 2002). In addition to RGD functionalization, QDs have been conjugated to several other peptides for tumor-specific targeting. Gao et al. designed and chemically synthesized anti-HER2 affibody molecules ( $Z$ HER2:342) for the specific targeting of HER2-expressing cells and tumors in imaging. Affibodies are small protein molecules that are engineered to specifically bind targets, such as other proteins or peptides. The synthesized Z HER2:342-QD conjugates exhibited tumor-targeting ability with superior imaging contrast in mice bearing HER2-overexpressing SKOV3 tumors compared to low-level HER2-expressing PC-3 mice. These results indicated that the Z HER2:342-QD conjugates were target-specific and had the potential to image HER2-overexpressing cells (Gao et al., 2011). Diagaradjane et al. prepared EGF-conjugated QDs that had the ability to distinguish EGF receptor-overexpressing tumors from nearby normal tissues. In vivo imaging exhibited three distinct phases for the EGF-QD nanoprobes: tumor influx ( $\sim 3 \mathrm{~min})$, clearance $(\sim 60 \mathrm{~min})$ and accumulation $(1-6 \mathrm{~h})$. The prepared EGF-QDs exhibited optimal pharmacokinetics and superior docking ability to EGFR, which allowed quantifiable imaging of EGFR expression in human colorectal cancer xenografts in mice (Diagaradjane et al., 2008).

Qian et al. employed Tf and anti-claudin 4-conjugated $\mathrm{CdSe} / \mathrm{CdS} / \mathrm{ZnS}$ QDs for targeted imaging of pancreatic cancer. The targeted optical agent was stable and had superior 
photoluminescence efficiency, demonstrating pancreatic cellspecific uptake using a monoclonal antibody for anti-claudin 4 . The targeting agent used in this study can be further tuned to image pancreatic cancer in its early stages (Qian et al., 2007). Yong et al. used anti-claudin 4- and anti-prostate stem cell antigen-bearing non-cadmium-based QDs (InP/ZnS QDs) as highly efficient, non-toxic targeted optical probes for imaging live pancreatic cancer cells. Antibodies, such as anti-claudin 4 and anti-prostate stem cell antigen, whose receptors are known to be upregulated in both primary and metastatic pancreatic cancers, were used as targeting agents. The targeted QDs demonstrated affinity and specific targeting for pancreatic cancer cell lines in vitro. In addition, the receptor-mediated delivery of targeted QDs was further confirmed by the observation of poor in vitro targeting in non-pancreatic cancer cell lines, which were negative for the claudin-4-receptor. These observations suggest that InP/ZnS QDs could be used as an efficient OI imaging nanoprobe in diagnostic imaging, particularly for early detection of pancreatic cancer (Yong et al., 2009).

\section{Clearance of QDs}

Choi et al. established the effect of hydrodynamic diameter (HD) and surface charge of QDs on their renal clearance. QDs with HD smaller than $5.5 \mathrm{~nm}$ demonstrated rapid and efficient urinary excretion. With the increase of HD from 4.36 to $8.65 \mathrm{~nm}$, the serum half-life increased from $48 \mathrm{~min}$ to $20 \mathrm{~h}$ (25 fold increase), suggesting a positive correlation between serum half-life and HD. QDs with HD range of 4.36-5.52 was cleared form the body through renal mechanism, while QDs with large HD did not undergo renal clearance and was confined to the liver, lung and spleen. The anionic or cationic surface charge of QDs increased the HD by $15 \mathrm{~nm}$ due to the charge related adsorption by serum proteins, which decreased the renal clearance consequently. Whereas, zwitterionic coatings prevented the serum protein adsorption and demonstrated increased solubility and improved renal filtration (Choi et al., 2007).

Fischer et al. investigated the clearance of two different QD conjugates [mercaptoundecanoic acid (QD-LM) and bovine serum albumin (QD-BSA)] administered intravenously in rats. QD-BSA exhibited accelerated clearance (1.23 \pm $0.22 \mathrm{mLmin}^{-1} \mathrm{~kg}^{-1}$ ) from the blood, which resulted in higher levels of liver uptake (99\%) compared to QD-LM (40\%). However, both conjugates were not detected in urine or feces during the entire time of experiment (10 days). This may be presumably due to large HD (25 nm for QD-LM and $80 \mathrm{~nm}$ for QD-BSA) (Fischer et al., 2006). Schipper et al. studied the clearance of two commercially available QDs with sizes 12 and $21 \mathrm{~nm}$ in diameter and effect of PEGylation on their clearance. The results showed that a $2000 \mathrm{~mW}$ PEG coating prolonged the blood half-life, but delayed accumulation in the liver. All particles were rapidly taken up and accumulated in the liver, whereas PEGylated particles accumulated in the bone, while unPEGylated particles did not (Schipper et al., 2007).

\section{GOLD NANOPARTICLES}

The popularity of gold nanoparticles (AuNPs) in biomedical applications has increased significantly in recent years owing to their unique size-dependent properties. AuNPs are highly biocompatible, easy to prepare and generally regarded as non-toxic (Boisselier and Astruc, 2009). Their characteristic surface plasmon absorption property is responsible for their excellent optical properties, making them attractive candidates for labeling and imaging. Tailor-made AuNPs of different sizes, shapes and functionalized with various targeting molecules have been used for a number of applications in cancer therapy and imaging (Tong et al., 2009; Kumar et al., 2013). Due to their vast potential, several AuNP-based formulations are currently undergoing phase I and phase II clinical trials for cancer treatment (Thakor et al., 2011).

The ability of AuNPs to quench fluorophores in close proximity encouraged Lee et al. to develop protease-sensitive NIRF probes for in vivo cancer imaging. In this study, AuNPs $(20 \mathrm{~nm})$ were stabilized by a Cy5.5-labeled MMP substrate, and the fluorescence of Cy5.5 was strongly quenched by the coordinated dye-dye and AuNP-surface quenching effects. When incubated with MMP in vitro, cleavage of the substrate allowed the probe to recover a strong fluorescence signal. In MMP-2-positive, SCC7 tumor-bearing mice, the administered probes demonstrated preferential tumor accumulation with a strong fluorescence signal (Lee et al., 2008). Popovtzer et al. developed gold nanorods (AuNRs) conjugated to UMA9 antibodies for the targeted CT imaging of head and neck cancers. SCC represents more than $90 \%$ of all head and neck cancers, and UMA9 antibody can target SCC tumors in vivo. In vitro CT imaging revealed that targeted AuNRs were readily taken up by A9 antigen-overexpressing cancer cells (UM-SCC-1 and UM-SCC-5), which showed strongly selective $\mathrm{X}$-ray attenuation that was distinct from that of normal cells (Popovtzer et al., 2008). Mu et al. developed AuNP-based activatable NIR probes functionalized with PEG and heterogeneous monolayers of dye-labeled peptides to monitor proteolytic activity in vivo. In this study, AuNPs (20 nm in diameter) were modified with a heterogeneous monolayer of fluorophore (Q670) and dark quencher (BHQ-2)-labeled peptide substrates that were responsive to proteases, such as trypsin and uPA (Figure 6). The resulting system demonstrated strong quenching properties due to the close proximity between Q670 and BHQ-2 to the AuNPs. In the presence of target enzymes in vitro, the nanoprobes recovered fluorescence with a five- to eight-fold increase in signal. In addition, AuNP-based probes exhibited prolonged circulation time in vivo with a $\mathrm{t}_{1 / 2}$ of more than $4 \mathrm{~h}$ and high image contrast in a mouse tumor model (Mu et al., 2010).

Chanda et al. developed bombesin (BBN) peptide-conjugated AuNPs to target gastrin-releasing peptide (GRP) receptors that are overexpressed on prostate cancer cells. BBN peptides exhibit a high affinity for GRP receptors that are overexpressed in prostate, breast and small-cell lung carcinomas in vivo. In vitro radioiodinated BBN displacement assays clearly demonstrated the GRP receptor specificity and binding affinity of the AuNP-BBN conjugates in PC-3 cells. In vivo CT imaging in prostate tumorbearing, severe combined immune deficiency mice showed that intra-peritoneally administered AuNP-BBN conjugates preferentially accumulated in the tumors with little or no uptake in the liver relative to controls (Chanda et al., 2010). Kim et al. used multifunctional aptamer-AuNP conjugates for the combined targeted CT imaging of and therapy for prostate cancer. AuNPs 


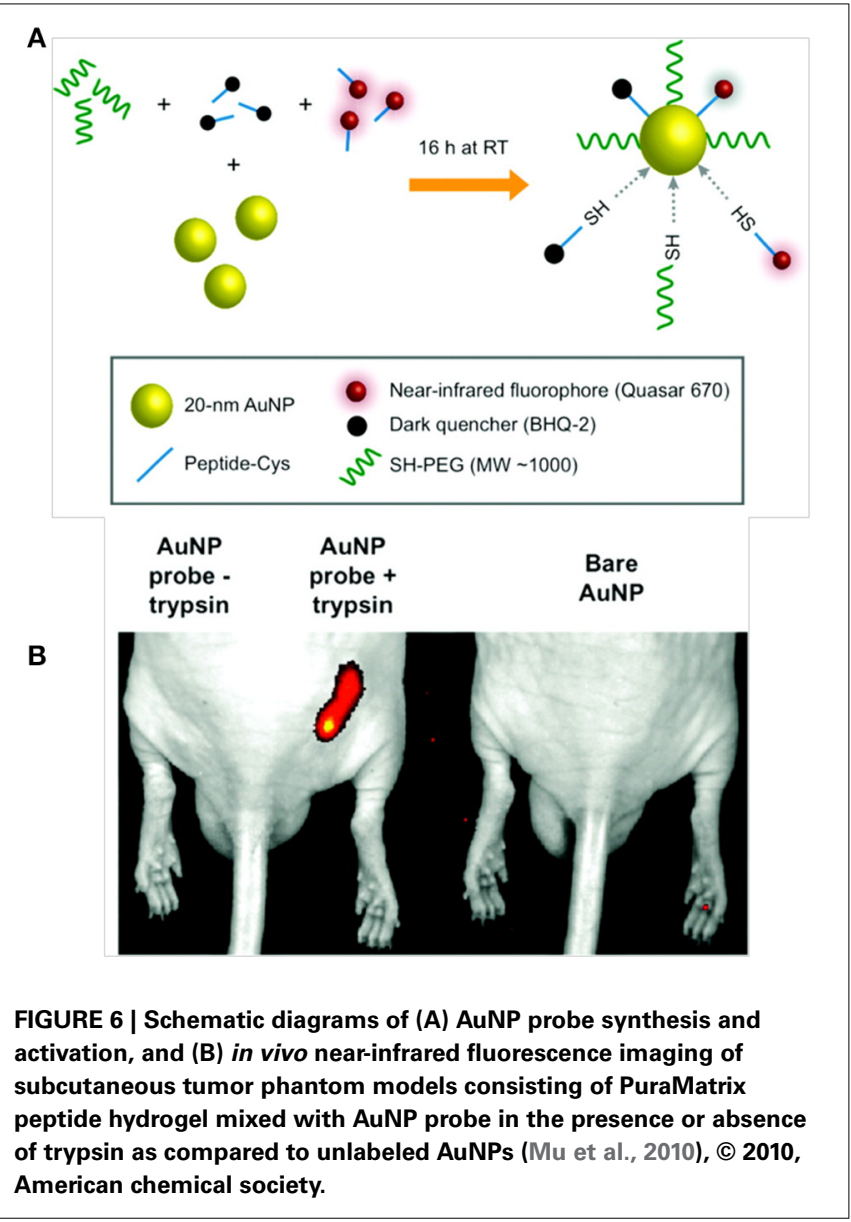

were coated with thiol-functionalized capture oligonucleotides (ONT) that consisted of an A10 spacer and a (TCG) ${ }_{7} \mathrm{ONT}$, which was hybridized with PSMA aptamer- $(\mathrm{CGA})_{7}$ to create a double-stranded GC region on which several DOX molecules could be loaded. The targeted PSMA aptamer-AuNPs exhibited more than four-fold greater CT intensity in LNCaP cells that overexpressed PSMA antigen than in PSMA-negative PC3 cells. In addition, drug-loaded, aptamer-AuNPs demonstrated targetspecific drug delivery to target cancer cells but not non-targeted cells (Kim et al., 2010). Chen et al. developed FA-conjugated bimodal G5 dendrimer nanoparticles carrying AuNPs loaded with Gd for targeted dual CT/MR tumor imaging. The multifunctional nanoprobes demonstrated the ability to image FA receptor overexpressing cancer models in vitro and in vivo (Chen et al., 2013).

Sun et al. reported tumor-targeting probes based on AuNPs for dual CT/OI of cancer. The targeted probe consisted of a Cy5.5-labeled, MMP-activatable peptide substrate that was chemically conjugated to glycol chitosan-coated AuNPs. The fluorescence of Cy5.5 was strongly quenched by BHQ3. However, when exposed to MMP-2, the quenched fluorescence was recovered by peptide cleavage. Following intravenous injection of the targeted AuNPs in mice bearing MMP-2-positive, HT-29 tumors, the targeted nanoparticles selectively accumulated in tumors, as observed by CT/fluorescence imaging (Sun et al.,
2011). Deng et al. developed molecular beacons based on rodshaped AuNPs for the targeted in vivo detection of matriptase expression on tumors. The molecular beacons consisted of an activatable fluorophore-peptide conjugate attached to AuNPs. The quenched fluorescence of the fluorophore by the AuNPs was restored when exposed to matriptase. After intratumoral injection of the targeted AuNPs in nude mice bearing HT-29 tumor xenografts with high matriptase expression, a strong fluorescence signal was observed in the tumors after $8 \mathrm{~h}$, clearly indicating the tumor-targeting ability of the AuNPs (Deng et al., 2013). Multifunctional nanoparticles based on AuNRs were developed for targeted positron emission tomography (PET) imaging of and therapy for cancer. The multifunctional nanoparticle system consisted of PEGylated AuNRs; cyclic RGD and a ${ }^{64} \mathrm{Cu}$-chelator were attached to the distal ends of PEG, and DOX was conjugated to the AuNRs via a linker that was vulnerable to tumor acidity. The AuNRs demonstrated target specificity in a relevant model both in vitro and in vivo. However, in U87MG tumor-bearing mice, PET imaging revealed similar biodistribution of both targeted and non-targeted AuNRs (Xiao et al., 2012). Lee et al. used heparinase-specific targeted AuNPs (AuNPHHep)-based activatable probes to detect and induce apoptosis in metastatic cancer cells. AuNP-HHep consisted of AuNPs conjugated to fluorophore-labeled heparin and RGD-bearing PEG. The fluorescence was quenched by the surface of the AuNPs and was recovered after exposure to the enzyme heparinase, which is known to be overexpressed in metastatic cancer cells. In addition, this targeted probe demonstrated highly specific apoptotic activity for cancer cells over-expressing $\alpha_{v} \beta_{3}$ on their membranes, revealing that the accumulation of heparin within the cells triggered apoptotic events. The probe employed in this study could be useful for both imaging and treatment of metastatic cancers (Lee et al., 2010b).

\section{Clearance of AuNPs}

AuNPs can be excreted by kidney or liver, however, substantial research efforts are needed to determine the factors that affect mode of excretion. Balasubramanian et al. investigated the biodistribution of AuNPs and found that $20 \mathrm{~nm}$ particles accumulated in the kidneys starting at 1 month with a decrease in Au levels in urine. It was explained that AuNPs may adsorb to the serum proteins or aggregate over time, which increased the particle size making them difficult to pass through glomerular membrane. Authors suggested that prolonged accumulation of AuNPs in the kidneys could damage the glomerular membrane and reduce the excretion (Balasubramanian et al., 2010). Zhang et al. investigated the biodistribution of two PEGylated AuNPs with sizes 20 and $80 \mathrm{~nm}$ in diameter. Authors found that size of NPs strongly influence the biodistribution. For instance, $20 \mathrm{~nm}$ AuNPs demonstrated prolonged blood circulation and reduced uptake by the liver and the spleen compared to 80-nm AuNPs. Importantly, 20-nm AuNPs also excreted from the body, suggesting the validity of AuNPs with size less than $20 \mathrm{~nm}$ in diameter for in vivo applications (Zhang et al., 2009).

In their work, Lipka et al. showed that PEG $(10 \mathrm{kDa})$ modified AuNPs with a HD of $31 \mathrm{~nm}$ showed the most clearance via 
the hepato-biliary pathway compared to PEG (750 Da) modified AuNPs with a HD of $21 \mathrm{~nm}$. In this study, AuNPs with PEG $(10 \mathrm{kDa})$ are the NPs with the greatest diameter and a study by Johnston et al. (2010) showed that $1.4 \mathrm{~nm}$ NPs exhibited higher hepato-biliary clearance compared to $18 \mathrm{~nm}$. From these results, authors suggested that bile excretion may be significantly dependent on particles PEGylation but not on their size (Lipka et al., 2010).

\section{POLYMERIC NANOPARTICLES}

In addition to metallic nanoparticles, the use of polymeric nanoparticles $(\mathrm{PN})$ as imaging probes have received significant attention due to their unique properties. PN-based imaging probes have several advantages over conventional low-molecularweight probes, including prolonged circulation time, improved stability, and target specificity, passivity to the immune system and little or no toxicity. In recent years, biological science in association with polymer science has yielded novel hybrid materials with sophisticated architectures for clinical imaging and therapy (Duncan, 2003; Mitra et al., 2005; Lee et al., 2010a; Park, 2012).

Scherer et al. developed NIR polymer-based proteolytic beacons (PB-M7NIR) for the in vivo detection and quantitation of MMP-7 activity. PB-M7NIR was a PEGylated PAMAM-G4 dendrimer that was covalently coupled to a Cy5.5-labeled peptide substrate [Cy5.5-ahx-RPLALWRS(ahx)C] that monitored MMP-7 activity and to a quencher (AF750). In vitro OI studies showed a five-fold increase in fluorescence. The intravenous administration of PB-M7NIR in mice allowed for selective tumor visualization and localization with two point two-fold improved signals in MMP7 SW480 tumors relative to MMP7-negative tumors (Scherer et al., 2008). Oslon et al. developed activatable cell-penetrating peptide (ACCP)-conjugated bimodal G5 PAMAM dendrimer nanoparticles (ACCPDs) labeled with Cy5, Gd, or both. Cellular uptake of these nanoparticles in tumors was four to fifteen-fold higher than that of bare ACPPs. With OI, residual tumors and metastases as small as $200 \mu \mathrm{m}$ were detected. ACCPDs were also used to visualize tumors during surgery (Olson et al., 2009). Successful surgical removal of tumors depends on the surgeon's ability to recognize and differentiate tumors from normal tissues; thus, it would be beneficial to objectively assess tumor margins while performing surgery. ACCPDs demonstrated superior ability to identify tumor tissue in tumorbearing mice during surgery relative to ACCPs. As a result, fewer residual cancer cells were left in the animals following surgery, and the animals showed prolonged tumor-free survival and overall survival relative to animals resected using traditional bright-field illumination (Nguyen et al., 2010). In another study, Galande et al. developed self-quenched, dendrimeric peptide-based probes using multiple antigenic peptide (MAP), which fluoresced only after enzymatic treatment. The peptide-based probe was developed on a solid support that consisted of a dipeptide cathepsin S substrate (Leu-Arg) and PEG chains on the dendritic arms. An NIR dye, CyTE777, was attached to the N-terminus of the peptide to establish a dye-dye self-quenching system. In vitro studies showed that in the presence of protease cathepsin $S$, the peptidebased probe demonstrated a more than seventy-fold increase in

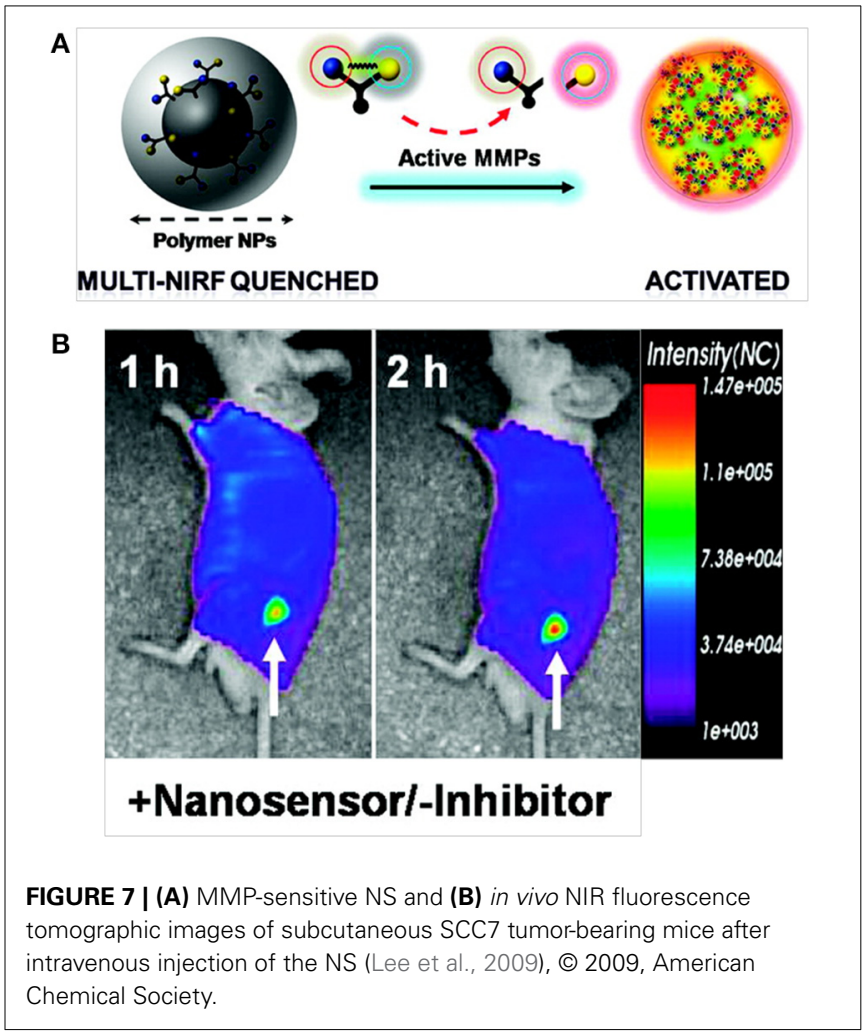

and more than 95\% recovery of fluorescence emission (Galande et al., 2006).

Lee et al. developed a self-assembled, chitosan nanoparticlebased nanosensor (NS) that consisted of activatable MMPspecific NIR fluorogenic peptides with an NIR dark quencher BHQ-3 (Figure 7). This type of peptide substrate-mediated fluorescence labeling of nanoparticles could be strongly dualquenched by both the dye-dark quencher and the NIR dye-dye self-quenching mechanisms. In the presence of MMP, the NIRdye substrate was cleaved, which resulted in NIR fluorescence signal recovery. Intravenously administered NSs demonstrated enzyme specificity in both MMP-positive, SCC-7 xenograft tumor and colon cancer mouse models (Lee et al., 2009). Kim et al. developed a polymeric nanoparticle-based probe that could sense early apoptosis. The probe was an on/off system that consisted of a caspase-3 cleavable, NIR dye peptide substrate (Cy5.5-DEVDC) covalently attached to a deoxycholic acidbearing polyethyleneamine conjugate. Due to the close proximity of Cy5.5 dyes to the nanoparticle surface, the fluorescence was self-quenched (off state). In the presence of caspase-3 enzyme in vitro, the nanoparticle recovered fluorescence (a ten-fold increase) that allowed monitoring of caspase-dependent apoptosis induced by tumor necrosis factor apoptosis inducing ligand in living HeLa cells (Kim et al., 2006).

\section{CLEARANCE OF PN}

Gref et al. studied the effect of PEG chain length (2 and $5 \mathrm{kDa}$ ) in preventing plasma protein adsorption on the surface of poly(lactic acid)-PEG (PLA-PEG) NPs. PLA-PEG (5 kDa) demonstrated minimal protein adsorption $(\sim 80 \%)$ compared 
Table 1 | Advantages and disadvantages of imaging modalities used with targeted or non-targeted imaging agents.

\begin{tabular}{|c|c|c|c|c|}
\hline Modality & Advantages & Disadvantages & Contrast agents & Radiation \\
\hline $\mathrm{CT}$ & $\begin{array}{l}\text { Unlimited tissue depth penetration } \\
\text { High spatial resolution } \\
\text { Can be used for whole body imaging } \\
\text { Low acquisition time } \\
\text { Anatomical imaging }\end{array}$ & $\begin{array}{l}\text { Radiation exposure } \\
\text { Poor soft tissue contrast }\end{array}$ & $\begin{array}{l}\text { Krypton } \\
\text { Xenon } \\
\text { lodine } \\
\text { Barium }\end{array}$ & X-rays \\
\hline $\begin{array}{l}\text { Positron } \\
\text { emission } \\
\text { tomography } \\
\text { (PET) }\end{array}$ & $\begin{array}{l}\text { Unlimited tissue depth penetration } \\
\text { Can be used for whole body imaging } \\
\text { Detailed anatomical information can be obtained } \\
\text { when combined with CT or MRI }\end{array}$ & $\begin{array}{l}\text { Radiation exposure } \\
\text { Low spatial resolution and } \\
\text { long acquisition time } \\
\text { Expensive }\end{array}$ & $\begin{array}{l}{ }^{11} \mathrm{C} \\
{ }^{18} \mathrm{~F} \\
{ }^{64} \mathrm{Cu}\end{array}$ & $\begin{array}{l}\text { High energy } \\
\gamma \text {-rays }\end{array}$ \\
\hline $\mathrm{MRI}$ & $\begin{array}{l}\text { Unlimited tissue depth penetration } \\
\text { Non-ionizing radiation } \\
\text { High spatial resolution and excellent soft tissue } \\
\text { contrast } \\
\text { Whole body imaging possible }\end{array}$ & $\begin{array}{l}\text { Expensive } \\
\text { Long acquisition time and } \\
\text { limited sensitivity }\end{array}$ & $\begin{array}{l}\mathrm{Gd}^{3+} \\
\text { SPIO, USPIO }\end{array}$ & Radio waves \\
\hline Ultrasound & $\begin{array}{l}\text { High spatial resolution } \\
\text { Real time imaging with low acquisition time } \\
\text { Highly sensitive and inexpensive }\end{array}$ & $\begin{array}{l}\text { Cannot be used for whole } \\
\text { body imaging }\end{array}$ & Microbubbles & $\begin{array}{l}\text { High frequency } \\
\text { sound }\end{array}$ \\
\hline Ol & $\begin{array}{l}\text { High spatial resolution } \\
\text { Real time imaging with low acquisition time } \\
\text { Highly sensitive and inexpensive }\end{array}$ & $\begin{array}{l}\text { Cannot be used for whole } \\
\text { body imaging } \\
\text { limited tissue depth } \\
\text { penetration }\end{array}$ & $\begin{array}{l}\text { Fluorescent dyes } \\
\text { and molecules } \\
\text { Light sensitive NPs }\end{array}$ & $\begin{array}{l}\text { Visible or } \\
\text { near-infrared } \\
\text { light }\end{array}$ \\
\hline
\end{tabular}

to non-PEGylated PLA NPs. PEG content between 2 and 5\% was determined as a threshold value for optimal protein resistance. Interestingly, distances of 1-2 nm between two terminally attached PEG chains were estimated for minimal protein adsorption (Gref et al., 2000). Using polystyrene NPs of two sizes 50 and $500 \mathrm{~nm}$, Kimura et al. studied the relation between protein adsorption kinetics and their liver uptake. $50 \mathrm{~nm}$ NPs exhibited rapid hepatic uptake when incubated with serum before transfusion in the rat liver. In vivo biodistribution of particle sizes of 50 and $500 \mathrm{~nm}$ revealed significant levels of agglomeration of larger particles in the liver. The surface charge of NPs has profound effect on uptake by the phagocytic system. Positively charged NPs are expected to have high non-specific internalization rate and short half-life compared to neutral or negatively charged NPs (Nagayama et al., 2007).

Kataoka et al. investigated the biodistribution of tyrosine (tyr) and tyrosine-glutamic acid (tyr-glu) conjugated PEG-poly (D, $\mathrm{L}^{-}$ lactide) micelles in mice. Both of these micelles [tyr (neutral) and tyr-glu (negative)] showed a significant long circulation time in the blood compartment with $25 \%$ of injected dose still circulating even at $24 \mathrm{~h}$. Biodistribution studies revealed that anionic micelle exhibited lower uptake into the liver and spleen compared to a neutral tyr-conjugated micelle, suggesting a substantial role of the anionic charge in avoiding non-specific organ uptake (Yamamoto et al., 2001).

\section{CONCLUSION AND PERSPECTIVES}

The current medical imaging technologies used in the clinic for cancer diagnosis and staging depend on anatomical changes. The advent of MI allows expression identification of specific biomolecules based on functional changes that are correlated with the genesis of cancer, which greatly enhances our understanding of disease mechanisms and progression. In addition, critical data obtained from MI would enable clinicians to plan and design treatments, which is crucial for patient compliance and survival. The development of targeted probes and optimizing their ability to reach a site of interest remains a herculean task. The inability of probes to cross various biological barriers largely accounts for their shortcomings. An ideal probe should essentially possess a high level of specificity for a target and should successfully overcome biological barriers.

Substantial developments in nanotechnology have resulted in the development of imaging probes functionalized with a diverse range of ligands. Due to the versatile nature of nanoparticles, creating custom-made probes with unique properties, such as superior targeting ability and enhanced in vivo half-life, is possible. Owing to these features, several multimodal imaging systems have been developed as powerful tools for accurate cancer diagnosis because they integrate more than one imaging technique. For instance, an MI probe that included NIR dye and MR contrast agents was developed and subsequently used for preoperative MRI and intraoperative brain tumor delineation through OI. Although nanoparticles have shown promising results as MI probes, proper safety evaluation regarding their toxicity is absolutely necessary before they enter clinical use.

\section{ACKNOWLEDGMENTS}

This work was supported by the NHRI Intramural research grants ME-103-pp-04 and NM-103-PP-01 from National Health Research Institutes of Taiwan. 


\section{REFERENCES}

Åkerman, M. E., Chan, W. C. W., Laakkonen, P., Bhatia, S. N., and Ruoslahti, E. (2002). Nanocrystal targeting in vivo. Proc. Natl. Acad. Sci. U.S.A. 99, 12617-12621. doi: 10.1073/pnas.152463399

Antony, A. C. (1996). Folate receptors. Ann. Rev. Nutr. 16, 501-521. doi: 10.1146/annurev.nu.16.070196.002441

Anzai, Y., Piccoli, C. W., Outwater, E. K., Stanford, W., Bluemke, D. A., Nurenberg, P., et al. (2003). Evaluation of neck and body metastases to nodes with Ferumoxtran 10-enhanced MR imaging: phase III safety and efficacy study. Radiology 228, 777-788. doi: 10.1148/radiol.2283020872

Bagalkot, V., Zhang, L., Levy-Nissenbaum, E., Jon, S., Kantoff, P. W., Langer, R., et al. (2007). Quantum Dot-Aptamer conjugates for synchronous cancer imaging, therapy, and sensing of drug delivery based on bi-fluorescence resonance energy transfer. Nano Lett. 7, 3065-3070. doi: 10.1021/nl071546n

Balasubramanian, S. K., Jittiwat, J., Manikandan, J., Ong, C. N., Yu, L. E., and Ong, W. Y. (2010). Biodistribution of gold nanoparticles and gene expression changes in the liver and spleen after intravenous administration in rats. Biomaterials 31, 2034-2042. doi: 10.1016/j.biomaterials.2009.11.079

Ballou, B., Fisher, G. W., Waggoner, A. S., Farkas, D. L., Reiland, J. M., Jaffe, R., et al. (1995). Tumor labeling in vivo using cyanine-conjugated monoclonal antibodies. Cancer Immunol. Immunother. 41, 257-263. doi: 10.1007/BF01 517001

Becker, A., Hessenius, C., Licha, K., Ebert, B., Sukowski, U., Semmler, W., et al. (2001). Receptor-targeted optical imaging of tumors with near-infrared fluorescent ligands. Nat. Biotechnol. 19, 327-331. doi: 10.1038/86707

Boisselier, E., and Astruc, D. (2009). Gold nanoparticles in nanomedicine: preparations, imaging, diagnostics, therapies and toxicity. Chem. Soc. Rev. 38, 1759-1782. doi: 10.1039/b806051g

Bourrinet, P., Bengele, H. H., Bonnemain, B., Dencausse, A., Idee, J. M., Jacobs, P. M., et al. (2006). Preclinical safety and pharmacokinetic profile of ferumoxtran10 , an ultrasmall superparamagnetic iron oxide magnetic resonance contrast agent. Invest. Radiol. 41, 313-324. doi: 10.1097/01.rli.0000197669. 80475.dd

Bruchez, M., Moronne, M., Gin, P., Weiss, S., and Alivisatos, A. P. (1998). Semiconductor nanocrystals as fluorescent biological labels. Science 281, 2013-2016. doi: 10.1126/science.281.5385.2013

Cai, W., Shin, D. W., Chen, K., Gheysens, O., Cao, Q., Wang, S. X., et al. (2006). Peptide-labeled near-infrared quantum dots for imaging tumor vasculature in living subjects. Nano Lett. 6, 669-676. doi: 10.1021/nl052405t

Caravan, P., Ellison, J. J., McMurry, T. J., and Lauffer, R. B. (1999). Gadolinium(III) chelates as MRI contrast agents: structure, dynamics, and applications. Chem. Rev. 99, 2293-2352. doi: 10.1021/cr980440x

Chanda, N., Kattumuri, V., Shukla, R., Zambre, A., Katti, K., Upendran, A., et al. (2010). Bombesin functionalized gold nanoparticles show in vitro and in vivo cancer receptor specificity. Proc. Natl. Acad. Sci. U.S.A. 107, 8760-8765. doi: $10.1073 /$ pnas. 1002143107

Chang, E., Miller, J. S., Sun, J., Yu, W. W., Colvin, V. L., Drezek, R., et al. (2005). Protease-activated quantum dot probes. Biochem. Biophy. Res. Commun 334, 1317-1321. doi: 10.1016/j.bbrc.2005.07.028

Chen, Q., Li, K., Wen, S., Liu, H., Peng, C., Cai, H., et al. (2013). Targeted CT/MR dual mode imaging of tumors using multifunctional dendrimer-entrapped gold nanoparticles. Biomaterials 34, 5200-5209. doi: 10.1016/j.biomaterials.2013.03.009

Chen, T. J., Cheng, T. H., Chen, C. Y., Hsu, S. C., Cheng, T. L., Liu, G. C., et al. (2009). Targeted Herceptin-dextran iron oxide nanoparticles for noninvasive imaging of HER2/neu receptors using MRI. J. Biol. Inorg. Chem. 14, 253-260. doi: 10.1007/s00775-008-0445-9

Cheng, K., and Cheng, Z. (2012). Near infrared receptor-targeted nanoprobes for early diagnosis of cancers. Curr. Med. Chem. 19, 4767-4785. doi: $10.2174 / 092986712803341458$

Cheng, W., Ping, Y., Zhang, Y., Chuang, K.-H., and Liu, Y. (2013). Magnetic resonance imaging (MRI) contrast agents for tumor diagnosis. J. Healthc. Eng. 4, 23-46. doi: 10.1260/2040-2295.4.1.23

Cheng, Z., Thorek, D. L. J., and Tsourkas, A. (2010). Gadolinium-conjugated dendrimer nanoclusters as a tumor-targeted T1 magnetic resonance imaging contrast agent. Angew. Chem. Int. Ed. 49, 346-350. doi: 10.1002/anie.2009 05133

Choi, H., Choi, S. R., Zhou, R., Kung, H. F., and Chen, I. W. (2004). Iron oxide nanoparticles as magnetic resonance contrast agent for tumor imaging via folate receptor-targeted delivery1. Acad. Radiol. 11, 996-1004. doi: 10.1016/j.acra.2004.04.018

Choi, H. S., Liu, W., Misra, P., Tanaka, E., Zimmer, J. P., Ipe, B. I., et al. (2007). Renal clearance of quantum dots. Nat. Biotechnol. 25, 1165-1170. doi: $10.1038 / \mathrm{nbt} 1340$

Choi, Y.-E., Kwak, J.-W., and Park, J. W. (2010). Nanotechnology for early cancer detection. Sensors 10, 428-455. doi: 10.3390/s100100428

Corot, C., Robert, P., Idée, J.-M., and Port, M. (2006). Recent advances in iron oxide nanocrystal technology for medical imaging. Adv. Drug Deliv. Rev. 58, 1471-1504. doi: 10.1016/j.addr.2006.09.013

Couvreur, P., and Vauthier, C. (2006). Nanotechnology: intelligent design to treat complex disease. Pharm. Res. 23, 1417-1450. doi: 10.1007/s11095-006-0284-8

Damadian, R. (1971). Tumor detection by nuclear magnetic resonance. Science 171, 1151-1153. doi: 10.1126/science.171.3976.1151

Deng, D., Zhang, D., Li, Y., Achilefu, S., and Gu, Y. (2013). Gold nanoparticles based molecular beacons for in vitro and in vivo detection of the matriptase expression on tumor. Biosens. Bioelectron. 49, 216-221. doi: 10.1016/j.bios.2013.05.018

Diagaradjane, P., Orenstein-Cardona, J. M., Colón-Casasnovas, N. E., Deorukhkar, A., Shentu, S., Kuno, N., et al. (2008). Imaging epidermal growth factor receptor expression in vivo: pharmacokinetic and biodistribution characterization of a bioconjugated quantum dot nanoprobe. Clin. Cancer Res. 14, 731-741. doi: 10.1158/1078-0432.CCR-07-1958

Duncan, R. (2003). The dawning era of polymer therapeutics. Nat. Rev. Drug Discov. 2, 347-360. doi: 10.1038/nrd1088

Enochs, W. S., Harsh, G., Hochberg, F., and Weissleder, R. (1999). Improved delineation of human brain tumors on MR images using a long-circulating, superparamagnetic iron oxide agent. J. Magn. Reson. Imaging 9, 228-232. doi: 10.1002/(SICI)1522-2586(199902)9:2<228::AID-JMRI12>3.0.CO;2-K

Fass, L. (2008). Imaging and cancer: a review. Mol. Oncol. 2, 115-152. doi: 10.1016/j.molonc.2008.04.001

Ferrari, M. (2005). Cancer nanotechnology: opportunities and challenges. Nat. Rev. Cancer 5, 161-171. doi: 10.1038/nrc1566

Fischer, H. C., Liu, L., Pang, K. S., and Chan, W. C. W. (2006). Pharmacokinetics of nanoscale quantum dots: in vivo distribution, sequestration, and clearance in the rat. Adv. Funct. Mater. 16, 1299-1305. doi: 10.1002/adfm.200500529

Galande, A., Hilderbrand, S., Weissleder, R., and Tung, C. (2006). Enzyme-targeted fluorescent imaging probes on a multiple antigenic peptide core. J. Med. Chem. 49, 4715-4720. doi: 10.1021/jm051001a

Gao, J., Chen, K., Miao, Z., Ren, G., Chen, X., Gambhir, S. S., et al. (2011). Affibodybased nanoprobes for HER2-expressing cell and tumor imaging. Biomaterials 32, 2141-2148. doi: 10.1016/j.biomaterials.2010.11.053

Gao, X., Cui, Y., Levenson, R. M., Chung, L. W. K., and Nie, S. (2004). In vivo cancer targeting and imaging with semiconductor quantum dots. Nat. Biotechnol. 22, 969-976. doi: 10.1038/nbt994

Gref, R., Lück, M., Quellec, P., Marchand, M., Dellacherie, E., Harnisch, S., et al. (2000). 'Stealth' corona-core nanoparticles surface modified by polyethylene glycol (PEG): influences of the corona (PEG chain length and surface density) and of the core composition on phagocytic uptake and plasma protein adsorption. Colloids Surf. B 18, 301-313. doi: 10.1016/S0927-7765(99) 00156-3

Han, B., Nakamura, M., Mori, I., Nakamura, Y., and Kakudo, K. (2005). Urokinase-type plasminogen activator system and breast cancer. Oncol. Rep. 14, 105-112. doi: 10.3892/or.14.1.105

Han, L., Huang, R., Li, J., Liu, S., Huang, S., and Jiang, C. (2011a). Plasmid pORF-hTRAIL and doxorubicin co-delivery targeting to tumor using peptideconjugated polyamidoamine dendrimer. Biomaterials 32, 1242-1252. doi: 10.1016/j.biomaterials.2010.09.070

Han, L., Huang, R., Liu, S., Huang, S., and Jiang, C. (2010). Peptide-conjugated PAMAM for targeted doxorubicin delivery to transferrin receptor overexpressed tumors. Mol. Pharm. 7, 2156-2165. doi: 10.1021/mp100185f

Han, L., Li, J., Huang, S., Huang, R., Liu, S., Hu, X., et al. (2011b). Peptideconjugated polyamidoamine dendrimer as a nanoscale tumor-targeted $\mathrm{T} 1$ magnetic resonance imaging contrast agent. Biomaterials 32, 2989-2998. doi: 10.1016/j.biomaterials.2011.01.005

Herbst, R. S. (2004). Review of epidermal growth factor receptor biology. Int. J. Radiat. Oncol. Biol. Phys. 59, S21-S26. doi: 10.1016/j.ijrobp.2003.11.041

Hopkins, C. R., and Trowbridge, I. S. (1983). Internalization and processing of transferrin and the transferrin receptor in human carcinoma A431 cells. J. Cell Biol. 97, 508-521. doi: 10.1083/jcb.97.2.508 
Huang, R., Han, L., Li, J., Liu, S., Shao, K., Kuang, Y., et al. (2011). Chlorotoxinmodified macromolecular contrast agent for MRI tumor diagnosis. Biomaterials 32, 5177-5186. doi: 10.1016/j.biomaterials.2011.03.075

Hussain, T., and Nguyen, Q. T. (2014). Molecular imaging for cancer diagnosis and surgery. Adv. Drug Deliv. Rev. 66, 90-100. doi: 10.1016/j.addr.2013.09.007

Islam, T., and Josephson, L. (2009). Current state and future applications of active targeting in malignancies using superparamagnetic iron oxide nanoparticles. Cance Biomark 5, 99-107. doi: 10.3233/CBM-2009-0615

Jacobs, R. E., and Cherry, S. R. (2001). Complementary emerging techniques: high-resolution PET and MRI. Curr. Opin. Neurobiol. 11, 621-629. doi: 10.1016/S0959-4388(00)00259-2

Jaffer, F. A., and Weissleder, R. (2005). MOlecular imaging in the clinical arena. JAMA 293, 855-862. doi: 10.1001/jama.293.7.855

Jain, T. K., Reddy, M. K., Morales, M. A., Leslie-Pelecky, D. L., and Labhasetwar, V. (2008). Biodistribution, clearance, and biocompatibility of iron oxide magnetic nanoparticles in rats. Mol. Pharm. 5, 316-327. doi: 10.1021/mp7001285

Jemal, A., Bray, F., Center, M. M., Ferlay, J., Ward, E., and Forman, D. (2011). Global cancer statistics. CA Cancer J. Clin. 61, 69-90. doi: 10.3322/caac.20107

Johnston, H. J., Semmler-Behnke, M., Brown, D. M., Kreyling, W., Tran, L., and Stone, V. (2010). Evaluating the uptake and intracellular fate of polystyrene nanoparticles by primary and hepatocyte cell lines in vitro. Toxicol. Appl. Pharmacol. 242, 66-78. doi: 10.1016/j.taap.2009.09.015

Juweid, M. E., and Cheson, B. D. (2006). Positron-emission tomography and assessment of cancer therapy. New Engl. J. Med. 354, 496-507. doi: 10.1056/NEJMra050276

Kelly, K. A., Setlur, S. R., Ross, R., Anbazhagan, R., Waterman, P., Rubin, M. A., et al. (2008). Detection of early prostate cancer using a hepsin-targeted imaging agent. Cancer Res. 68, 2286-2291. doi: 10.1158/0008-5472.CAN-07-1349

Kim, D., Jeong, Y. Y., and Jon, S. (2010). A drug-loaded Aptamer-Gold nanoparticle bioconjugate for combined CT imaging and therapy of prostate cancer. ACS Nano 4, 3689-3696. doi: 10.1021/nn901877h

Kim, K., Lee, M., Park, H., Kim, J. H., Kim, S., Chung, H., et al. (2006). CellPermeable and Biocompatible Polymeric Nanoparticles for Apoptosis Imaging. J. Am. Chem. Soc. 128, 3490-3491. doi: 10.1021/ja057712f

Kobayashi, H., and Brechbiel, M. (2005). Nano-sized MRI contrast agents with dendrimer cores. Adv. Drug Deliv. Rev. 57, 2271-2286. doi: 10.1016/j.addr.2005.09.016

Kobayashi, H., and Brechbiel, M. W. (2003). Dendrimer-based macromolecular MRI contrast agents: characteristics and application. Mol. Imaging 2, 1-10. doi: $10.1162 / 153535003765276237$

Kresse, M., Wagner, S., Pfefferer, D., Lawaczeck, R., Elste, V., and Semmler, W. (1998). Targeting of ultrasmall superparamagnetic iron oxide (USPIO) particles to tumor cells in vivo by using transferrin receptor pathways. Magn. Reson. Med. 40, 236-242. doi: 10.1002/mrm.1910400209

Kumar, A., Zhang, X., and Liang, X.-J. (2013). Gold nanoparticles: emerging paradigm for targeted drug delivery system. Biotechnol. Adv. 31, 593-606. doi: 10.1016/j.biotechadv.2012.10.002

LaVan, D. A., McGuire, T., and Langer, R. (2003). Small-scale systems for in vivo drug delivery. Nat. Biotechnol. 21, 1184-1191. doi: 10.1038/nbt876

Lee, K., Lee, H., Bae, K. H., and Park, T. G. (2010b). Heparin immobilized gold nanoparticles for targeted detection and apoptotic death of metastatic cancer cells. Biomaterials 31, 6530-6536. doi: 10.1016/j.biomaterials.2010. 04.046

Lee, S., Cha, E. J., Park, K., Lee, S. Y., Hong, J. K., Sun, I. C., et al. (2008). A nearinfrared-fluorescence-quenched gold-nanoparticle imaging probe for in vivo drug screening and protease activity determination. Angew. Chem. Int. Ed. 120, 2846-2849. doi: 10.1002/ange.200705240

Lee, S. H., Kim, B. H., Na, H. B., and Hyeon, T. (2014). Paramagnetic inorganic nanoparticles as T1MRI contrast agents. Wiley Interdiscip. Rev. Nanomed. Nanobiotechnol. 6, 196-209. doi: 10.1002/wnan.1243

Lee, S., Ryu, J. H., Park, K., Lee, A., Lee, S. Y., Youn, I. C., et al. (2009). Polymeric nanoparticle-based activatable near-infrared nanosensor for protease determination in vivo. Nano Lett. 9, 4412-4416. doi: 10.1021/nl9 $02709 \mathrm{~m}$

Lee, S., Xie, J., and Chen, X. (2010a). Activatable molecular probes for cancer imaging. Curr. Top. Med. Chem. 10, 1135-1144. doi: 10.2174/1568026107913 84270

Leuschner, C., Kumar, Challa, S. S. R., Hansel, W., and Hormes, J. (2005). Targeting breast cancer cells and their metastases through luteinizing hormone releasing hormone (LHRH) receptors using magnetic nanoparticles. J. Biomed. Nanotechnol. 1, 229-233. doi: 10.1166/jbn.2005.027

Li, X., Du, X., Huo, T., Liu, X., Zhang, S., and Yuan, F. (2009). Specific targeting of breast tumor by octreotide-conjugated ultrasmall superparamagnetic iron oxide particles using a clinical 3. 0-tesla magnetic resonance scanner. Acta. Radiol. 50, 583-594. doi: 10.1080/02841850902902557

Lin, W., Hyeon, T., Lanza, G. M., Zhang, M., and Meade, T. J. (2009). Magnetic nanoparticles for early detection of cancer by magnetic resonance imaging. MRS Bull. 34, 441-448. doi: 10.1557/mrs2009.120

Lipka, J., Semmler-Behnke, M., Sperling, R. A., Wenk, A., Takenaka, S., Schleh, C., et al. (2010). Biodistribution of PEG-modified gold nanoparticles following intratracheal instillation and intravenous injection. Biomaterials 31, 6574-6581. doi: 10.1016/j.biomaterials.2010.05.009

Lu, Y., and Low, P. S. (2002). Folate-mediated delivery of macromolecular anticancer therapeutic agents. Adv. Drug Deliv. Rev. 54, 675-693. doi: 10.1016/S0169-409X(02)00042-X

Luker, G. D., and Luker, K. E. (2008). Optical imaging: current applications and future directions. J. Nucl. Med. 49, 1-4. doi: 10.2967/jnumed.107.045799

Luo, K., Liu, G., She, W., Wang, Q., Wang, G., He, B., et al. (2011). Gadoliniumlabeled peptide dendrimers with controlled structures as potential magnetic resonance imaging contrast agents. Biomaterials 32, 7951-7960. doi: 10.1016/j.biomaterials.2011.07.006

Martin, S. J., Reutelingsperger, C. P., McGahon, A. J., Rader, J. A., van Schie, R. C., LaFace, D. M., et al. (1995). Early redistribution of plasma membrane phosphatidylserine is a general feature of apoptosis regardless of the initiating stimulus: inhibition by overexpression of Bcl-2 and Abl. J. Exp. Med. 182, 1545-1556. doi: 10.1084/jem.182.5.1545

Massoud, T. F., and Gambhir, S. S. (2003). Molecular imaging in living subjects: seeing fundamental biological processes in a new light. Gene. Dev. 17, 545-580. doi: $10.1101 / \operatorname{gad} .1047403$

McCarthy, J. R., Bhaumik, J., Karver, M. R., Sibel Erdem, S., and Weissleder, R. (2010). Targeted nanoagents for the detection of cancers. Mol. Oncol. 4, 511-528. doi: 10.1016/j.molonc.2010.08.003

McCarthy, J. R., and Weissleder, R. (2008). Multifunctional magnetic nanoparticles for targeted imaging and therapy. Adv. Drug Deliv. Rev. 60, 1241-1251. doi: 10.1016/j.addr.2008.03.014

Medintz, I. L., Uyeda, H. T., Goldman, E. R., and Mattoussi, H. (2005). Quantum dot bioconjugates for imaging, labelling and sensing. Nat. Mater. 4, 435-446. doi: $10.1038 /$ nmat 1390

Meng, J., Fan, J., Galiana, G., Branca, R. T., Clasen, P. L., Mad, S., et al. (2009). LHRH-functionalized superparamagnetic iron oxide nanoparticles for breast cancer targeting and contrast enhancement in MRI. Mater. Sci. Eng. C 29, 1467-1479. doi: 10.1016/j.msec.2008.09.039

Meng, X. X., Wan, J. Q., Jing, M., Zhao, S. G., Cai, W., Liu, E. Z., et al. (2007). Specific targeting of gliomas with multifunctional superparamagnetic iron oxide nanoparticle optical and magnetic resonance imaging contrast agents1. Acta Pharmacol. Sin. 28, 2019-2026. doi: 10.1111/j.1745-7254.2007. 00661.x

Michalet, X., Pinaud, F. F., Bentolila, L. A., Tsay, J. M., Doose, S., Li, J. J., et al. (2005). Quantum dots for live cells, in vivo imaging, and diagnostics. Science 307, 538-544. doi: 10.1126/science.1104274

Mitra, A., Mulholland, J., Nan, A., McNeill, E., Ghandehari, H., and Line, B. R. (2005). Targeting tumor angiogenic vasculature using polymer-RGD conjugates. J. Control. Release 102, 191-201. doi: 10.1016/j.jconrel.2004.09.023

Morgan, S., Grootendorst, P., Lexchin, J., Cunningham, C., and Greyson, D. (2011). The cost of drug development: a systematic review. Health Policy 100, 4-17. doi: 10.1016/j.healthpol.2010.12.002

Mu, C. J., LaVan, D. A., Langer, R. S., and Zetter, B. R. (2010). Self-assembled gold nanoparticle molecular probes for detecting proteolytic activity in vivo. ACS Nano 4, 1511-1520. doi: 10.1021/nn9017334

Mulder, W. J., Koole, R., Brandwijk, R. J., Storm, G., Chin, P. T., Strijkers, G. J., et al. (2005). Quantum dots with a paramagnetic coating as a bimodal molecular imaging probe. Nano Lett. 6, 1-6. doi: 10.1021/nl051935m

Nagayama, S., Ogawara, K.-I., Fukuoka, Y., Higaki, K., and Kimura, T. (2007) Time-dependent changes in opsonin amount associated on nanoparticles alter their hepatic uptake characteristics. Int. J. Pharm. 342, 215-221. doi: 10.1016/j.ijpharm.2007.04.036

Neuwelt, E. A., Várallyay, P., Bagó, A. G., Muldoon, L. L., Nesbit, G., and Nixon, R. (2004). Imaging of iron oxide nanoparticles by MR and light microscopy 
in patients with malignant brain tumours. Neuropathol. Appl. Neurobiol. 30, 456-471. doi: 10.1111/j.1365-2990.2004.00557.x

Nguyen, Q., Olson, E. S., Aguilera, T. A., Jiang, T., Scadeng, M., Ellies, L. G., et al. (2010). Surgery with molecular fluorescence imaging using activatable cellpenetrating peptides decreases residual cancer and improves survival. Proc. Natl. Acad. Sci. U.S.A. 107, 4317-4322. doi: 10.1073/pnas.0910261107

Olson, E. S., Aguilera, T. A., Jiang, T., Ellies, L. G., Nguyen, Q. T., Wong, E. H., et al. (2009). In vivo characterization of activatable cell penetrating peptides for targeting protease activity in cancer. Integr. Biol. 1, 382-393. doi: 10.1039/b904890a

Olson, E. S., Jiang, T., Aguilera, T. A., Nguyen, Q. T., Ellies, L. G., Scadeng, M., et al. (2010). Activatable cell penetrating peptides linked to nanoparticles as dual probes for in vivo fluorescence and MR imaging of proteases. Proc. Natl. Acad. Sci. U.S.A. 107, 4311-4316. doi: 10.1073/pnas.0910283107

Park, K. (2012). Polysaccharide-based near-infrared fluorescence nanoprobes for cancer diagnosis. Quant. Imaging Med. Surg. 2, 106-113. doi: 10.3978/j.issn. 2223-4292.2012.05.01

Peer, D., Karp, J. M., Hong, S., Farokhzad, O. C., Margalit, R., and Langer, R. (2007) Nanocarriers as an emerging platform for cancer therapy. Nat. Nanotechnol. 2, 751-760. doi: 10.1038/nnano.2007.387

Pericleous, P., Gazouli, M., Lyberopoulou, A., Rizos, S., Nikiteas, N., and Efstathopoulos, E. P. (2012). Quantum dots hold promise for early cancer imaging and detection. Int. J. Cancer 131, 519-528. doi: 10.1002/ijc.27528

Pham, W., Choi, Y., Weissleder, R., and Tung, C.-H. (2004). Developing a PeptideBased Near-Infrared Molecular Probe for Protease Sensing. Bioconjug. Chem 15 1403-1407. doi: 10.1021/bc049924s

Pilch, J., Brown, D. M., Komatsu, M., Järvinen, T. A., Yang, M., Peters, D., et al. (2006). Peptides selected for binding to clotted plasma accumulate in tumor stroma and wounds. Proc. Natl. Acad. Sci. U.S.A. 103, 2800-2804. doi: 10.1073/pnas.0511219103

Popovtzer, R., Agrawal, A., Kotov, N. A., Popovtzer, A., Balter, J., Carey, T. E., et al. (2008). Targeted gold nanoparticles enable molecular CT imaging of cancer. Nano Lett. 8, 4593-4596. doi: 10.1021/nl8029114

Pysz, M. A., Gambhir, S. S., and Willmann, J. K. (2010). Molecular imaging: current status and emerging strategies. Clin. Radiol. 65, 500-516. doi: 10.1016/j.crad.2010.03.011

Qian, J., Yong, K. T., Roy, I., Ohulchanskyy, T. Y., Bergey, E. J., Lee, H. H., et al. (2007). Imaging pancreatic cancer using surface-functionalized quantum dots. J. Phys. Chem. B 111, 6969-6972. doi: 10.1021/jp070620n

Regino, C. A., Walbridge, S., Bernardo, M., Wong, K. J., Johnson, D., Lonser, R. et al. (2008). A dual CT-MR dendrimer contrast agent as a surrogate marker for convection-enhanced delivery of intracerebral macromolecular therapeutic agents. Contrast Media Mol. Imaging 3, 2-8. doi: 10.1002/cmmi.223

Rudin, M., and Weissleder, R. (2003). Molecular imaging in drug discovery and development. Nat. Rev. Drug Discov. 2, 123-131. doi: 10.1038/nrd1007

Saini, S., Stark, D. D., Hahn, P. F., Wittenberg, J., Brady, T. J., and Ferrucci, J. T. Jr. (1987). Ferrite particles: a superparamagnetic MR contrast agent for the reticuloendothelial system. Radiology 162, 211-216. doi: 10.1148/radiology.162.1.3786765

Saravanakumar, G., Kim, K., Park, J. H., Rhee, K., and Kwon, I. C. (2009). Current status of nanoparticle-based imaging agents for early diagnosis of cancer and atherosclerosis. J. Biomed. Nanotechnol. 5, 20-35. doi: 10.1166/jbn.2009.030

Sarin, H., Kanevsky, A. S., Wu, H., Brimacombe, K. R., Fung, S. H., Sousa, A. A., et al. (2008). Effective transvascular delivery of nanoparticles across the blood-brain tumor barrier into malignant glioma cells. J. Transl. Med. 6:80. doi: 10.1186/1479-5876-6-80

Schellenberger, E. A., Bogdanov, A. Jr., Högemann, D., Tait, J., Weissleder, R., and Josephson, L. (2002). Annexin V-CLIO: a nanoparticle for detecting apoptosis by MRI. Mol. Imaging 1, 102-107. doi: 10.1162/153535002320162769

Scherer, R. L., VanSaun, M. N., McIntyre, J. O., and Matrisian, L. M. (2008). Optical imaging of matrix metalloproteinase-7 activity in vivo using a proteolytic nanobeacon. Mol. Imaging 7, 118-1131. doi: 10.2310/7290.2008.00010

Schipper, M. L., Cheng, Z., Lee, S. W., Bentolila, L. A., Iyer, G., Rao, J., et al. (2007). microPET-based biodistribution of quantum dots in living mice. J. Nucl. Med. 48, 1511-1518. doi: 10.2967/jnumed.107.040071

Schnell, O., Krebs, B., Carlsen, J., Miederer, I., Goetz, C., Goldbrunner, R. H., et al. (2009). Imaging of integrin $\alpha v \beta 3$ expression in patients with malignant glioma by [18F] Galacto-RGD positron emission tomography. Neuro Oncol. 11, 861-870. doi: 10.1215/15228517-2009-024
Schroeder, J. E., Shweky, I., Shmeeda, H., Banin, U., and Gabizon, A. (2007). Folatemediated tumor cell uptake of quantum dots entrapped in lipid nanoparticles. J. Control. Release 124, 28-34. doi: 10.1016/j.jconrel.2007.08.028

Siegel, R., Ma, J., Zou, Z., and Jemal, A. (2014). Cancer statistics, 2014. CA Cancer J. Clin. 64, 9-29. doi: 10.3322/caac. 21208

Smith, B. R., Cheng, Z., De, A., Koh, A. L., Sinclair, R., and Gambhir, S. S. (2008). Real-time intravital imaging of RGD-quantum dot binding to luminal endothelium in mouse tumor neovasculature. Nano Lett. 8, 2599-2606. doi: $10.1021 /$ nl080141f

Soroceanu, L., Gillespie, Y., Khazaeli, M. B., and Sontheimer, H. (1998). Use of chlorotoxin for targeting of primary brain tumors. Cancer Res. 58, 4871-4879.

Sosnovik, D. E., and Weissleder, R. (2007). Emerging concepts in molecular MRI. Curr. Opin. Biotechnol. 18, 4-10. doi: 10.1016/j.copbio.2006.11.001

Stark, D. D., and Bradley, W. J. Jr. (1999). Magnetic Resonance Imaging. St. Louis, MO: Mosby.

Sun, C., Veiseh, O., Gunn, J., Fang, C., Hansen, S., Lee, D., et al. (2008). In vivo MRI detection of gliomas by chlorotoxin-conjugated superparamagnetic nanoprobes. Small 4, 372-379. doi: 10.1002/smll.200700784

Sun, I. C., Eun, D. K., Koo, H., Ko, C. Y., Kim, H. S., Yi, D. K., et al. (2011). Tumortargeting gold particles for dual computed tomography/optical cancer imaging. Angew. Chem. Int. Ed. 50, 9348-9351. doi: 10.1002/anie.201102892

Swanson, S. D., Kukowska-Latallo, J. F., Patri, A. K., Chen, C., Ge, S., Cao, Z et al. (2008). Targeted gadolinium-loaded dendrimer nanoparticles for tumorspecific magnetic resonance contrast enhancement. Int. J. Nanomedicine 3, 201-210. doi: 10.2147/IJN.S2696

Tan, M., Ye, Z., Lindner, D., Brady-Kalnay, S., and Lu, Z.-R. (2014). Synthesis and evaluation of a targeted nanoglobular dual-modal imaging agent for MR Imaging and image-guided surgery of prostate cancer. Pharm. Res. 31, 1-8. doi: 10.1007/s11095-013-1008-5

Thakor, A. S., Jokerst, J., Zavaleta, C., Massoud, T. F., and Gambhir, S. S. (2011) Gold nanoparticles: a revival in precious metal administration to patients. Nano Lett. 11, 4029-4036. doi: 10.1021/nl202559p

Tong, L., Wei, Q., Wei, A., and Cheng, J.-X. (2009). Gold nanorods as contrast agents for biological imaging: optical properties, surface conjugation and photothermal effects. Photochem. Photobiol. 85, 21-32. doi: 10.1111/j.17511097.2008.00507.x

Tran, T., Engfeldt, T., Orlova, A., Sandström, M., Feldwisch, J., Abrahmsén, L., et al. (2007). 99mTc-maEEE-ZHER2:342, an affibody molecule-based tracer for the detection of HER2 expression in malignant tumors. Bioconjug. Chem. 18, 1956-1964. doi: 10.1021/bc7002617

Weissleder, R. (2002). Scaling down imaging: molecular mapping of cancer in mice. Nat. Rev. Cancer 2, 11-18. doi: 10.1038/nrc701

Weissleder, R. (2006). Molecular imaging in cancer. Science 312, 1168-1171. doi: $10.1126 /$ science.1125949

Weissleder, R., and Pittet, M. J. (2008). Imaging in the era of molecular oncology. Nature 452, 580-589. doi: 10.1038/nature06917

Willmann, J. K., van Bruggen, N., Dinkelborg, L. M., and Gambhir, S. S. (2008). Molecular imaging in drug development. Nat. Rev. Drug Discov. 7, 591-607. doi: $10.1038 / \mathrm{nrd} 2290$

Xiao, Y., Hong, H., Matson, V. Z., Javadi, A., Xu, W., Yang, Y., et al. (2012). Gold Nanorods conjugated with doxorubicin and cRGD for combined anticancer drug delivery and PET imaging. Theranostics 2, 757-768. doi: $10.7150 /$ thno. 4756

Xu, H., Regino, C. A., Koyama, Y., Hama, Y., Gunn, A. J., Bernardo, M., et al. (2007). Preparation and preliminary evaluation of a biotin-targeted, lectin-targeted dendrimer-based probe for dual-modality magnetic resonance and fluorescence imaging. Bioconj. Chem 18, 1474-1482. doi: 10.1021/bc0701085

Yamamoto, Y., Nagasaki, Y., Kato, Y., Sugiyama, Y., and Kataoka, K. (2001). Long-circulating poly(ethylene glycol)-poly(d,l-lactide) block copolymer micelles with modulated surface charge. J. Control. Release 77, 27-38. doi: 10.1016/S0168-3659(01)00451-5

Yang, L., Cao, Z., Sajja, H. K., Mao, H., Wang, L., Geng, H., et al. (2008). Development of receptor targeted magnetic iron oxide nanoparticles for efficient drug delivery and tumor imaging. J. Biomed. Nanotechnol. 4, 439-449. doi: 10.1166/jbn.2008.007

Yang, L., Mao, H., Wang, Y. A., Cao, Z., Peng, X., Wang, X., et al. (2009b). Single chain epidermal growth factor receptor antibody conjugated nanoparticles for in vivo tumor targeting and imaging. Small 5, 235-243. doi: $10.1002 / \mathrm{smll} .200800714$ 
Yang, L., Peng, X. H., Wang, Y. A., Wang, X., Cao, Z., Ni, C., et al. (2009a). Receptortargeted nanoparticles for in vivo imaging of breast cancer. Clin. Cancer Res. 15, 4722-4732. doi: 10.1158/1078-0432.CCR-08-3289

Yong, K. T., Ding, H., Roy, I., Law, W. C., Bergey, E. J., Maitra, A., et al. (2009). Imaging pancreatic cancer using bioconjugated InP quantum dots. ACS Nano 3, 502-510. doi: 10.1021/nn8008933

Zhang, G., Yang, Z., Lu, W., Zhang, R., Huang, Q., Tian, M., et al. (2009). Influence of anchoring ligands and particle size on the colloidal stability and in vivo biodistribution of polyethylene glycol-coated gold nanoparticles in tumor-xenografted mice. Biomaterials 30, 1928-1936. doi: 10.1016/j.biomaterials.2008.12.038

Zhang, H., Yee, D., and Wang, C. (2008). Quantum dots for cancer diagnosis and therapy: biological and clinical perspectives. Nanomedicine 3, 83-91. doi: $10.2217 / 17435889.3 .1 .83$

Zhou, Z., and Lu, Z.-R. (2013). Gadolinium-based contrast agents for magnetic resonance cancer imaging. Wiley Interdiscip. Rev. Nanomed. Nanobiotechnol. 5, 1-18. doi: 10.1002/wnan.1198
Conflict of Interest Statement: The authors declare that the research was conducted in the absence of any commercial or financial relationships that could be construed as a potential conflict of interest.

Received: 14 July 2014; accepted: 19 September 2014; published online: 17 October 2014.

Citation: Sivasubramanian M, Hsia $Y$ and Lo L-W (2014) Nanoparticle-facilitated functional and molecular imaging for the early detection of cancer. Front. Mol. Biosci. 1:15. doi: 10.3389/fmolb.2014.00015

This article was submitted to Molecular Diagnostics, a section of the journal Frontiers in Molecular Biosciences.

Copyright (c) 2014 Sivasubramanian, Hsia and Lo. This is an open-access article distributed under the terms of the Creative Commons Attribution License (CC BY). The use, distribution or reproduction in other forums is permitted, provided the original author(s) or licensor are credited and that the original publication in this journal is cited, in accordance with accepted academic practice. No use, distribution or reproduction is permitted which does not comply with these terms. 